\title{
Bioprinted 3D Outer Retina Barrier Uncovers RPE- dependent Choroidal Phenotype in Advanced Macular Degeneration
}

\section{Kapil Bharti ( $\square$ kapil.bharti@nih.gov )}

Ocular and Stem Cell Translational Research Section, National Eye Institute, National Institutes of Health, Bethesda, MD 20892

\section{Min Jae Song}

National Center for Advancing Translational Sciences, National Eye Institute, National Institutes of Health, National Institutes of Health

\section{Russell Quinn}

National Eye Institute, National Institutes of Health

\section{Eric Nguyen}

National Eye Institute, National Institutes of Health https://orcid.org/0000-0002-4164-5548

\section{Tea Soon Park}

National Eye Institute, National Institutes of Health

\section{Christopher Hampton}

National Eye Institute, National Institutes of Health

\section{Ruchi Sharma}

Unit on Ocular and Stem Cell Translational Research, National Eye Institute, National Institutes of Health

\section{Céline Koster}

Department of Clinical Genetics, Amsterdam University Medical Centers (AUMC), Location Academic Medical Center (AMC), University of Amsterdam (UvA)

\section{Ty Voss}

National Center for Advancing Translational Sciences

\section{Carlos Tristan}

National Center for Advancing Translational Sciences

\section{Claire Malley}

$\mathrm{NIH}$ National Center for Advancing Translational Sciences https://orcid.org/0000-0002-2352-8479

\section{Anju Singh}

National Center for Advancing Translational Sciences, National Institutes of Health

\section{Roba Dejene}

National Eye Institute, National Institutes of Health

\section{Devika Bose}

National Eye Institute, National Institutes of Health 


\section{Paige Derr}

$\mathrm{NIH}$

\section{Kristy Derr}

$\mathrm{NIH}$

\section{Sam Michael}

National Center for Advancing Translational Sciences

\section{Francesca Barone}

National Eye Institute, National Institutes of Health

Arvydas Maminishkis

National Eye Institute, National Institutes of Health

llyas Singec

NCATS/NIH

Marc Ferrer

NCATS, NIH

\section{Article}

Keywords:

Posted Date: January 13th, 2021

DOl: https://doi.org/10.21203/rs.3.rs-135775/v1

License: (c) (i) This work is licensed under a Creative Commons Attribution 4.0 International License. Read Full License 

Macular Degeneration

3 Min Jae Song ${ }^{1,2}$, Russ Quinn ${ }^{1}$, Eric Nguyen ${ }^{1}$, Christopher Hampton ${ }^{1}$, Ruchi Sharma ${ }^{1}$, Tea Soon Park ${ }^{1}$,

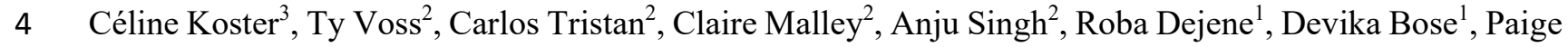

5 Derr $^{2}$, Kristy Derr ${ }^{2}$, Sam Michael ${ }^{2}$, Francesca Barone ${ }^{1}$, Arvydas Maminishkis ${ }^{1}$, Ilyas Singec ${ }^{2}$, Marc Ferrer ${ }^{2}$, 6 Kapil Bharti*1,2

${ }^{1}$ National Eye Institute, National Institutes of Health

${ }^{2}$ National Center for Advancing Translational Sciences, National Institutes of Health

${ }^{3}$ Department of Clinical Genetics, Amsterdam University Medical Centers (AUMC), Location Academic Medical Center (AMC), University of Amsterdam (UvA), 1105 AZ Amsterdam, The Netherlands.

12 Keywords: Age-related macular degeneration, choroidal neovascularization, engineered vascular-outerblood-retina-barrier, retinal pigment epithelium, Bruch's membrane, choriocapillaris 


\section{Abstract}

25 Age-related macular degeneration (AMD), a leading cause of blindness, initiates in the outer-blood-retina26 barrier (oBRB) formed by Retinal pigment epithelium (RPE), Bruch's membrane, and choriocapillaris. The

27 mechanism of AMD initiation and progression remain poorly understood due to the lack of physiologically 28 relevant oBRB models. We engineered a native-like 3D-oBRB tissue by bioprinting endothelial cells, 29 pericytes, and fibroblasts on the basal side of a biodegradable scaffold and establishing an RPE monolayer 30 on top. In this 3D-oBRB, a fully-polarized RPE monolayer with apical processes and basal infoldings 31 provides barrier resistance, induces fenestration and choroid-specific gene expression in the

32 choriocapillaris, and supports the formation of a Bruch's-like membrane that allows tissue integration in 33 rat eyes. Complement activation in the 3D-oBRB triggers dry-AMD phenotypes (including subRPE drusen 34 and choriocapillaris degeneration), and hypoxia activated HIF- $\alpha$ induces wet-AMD phenotypes 35 (choriocapillaris neovascularization). Anti-VEGF drug treatment suppresses neovascularization 36 validating this model for clinical translation and drug discovery. 
47 Age-related macular degeneration (AMD) affects over 196 million people worldwide and leads to blindness 48 in advanced stages ${ }^{1}$. Atrophy of the retinal pigment epithelium (RPE) and the choriocapillaris of the choroid 49 in advanced AMD stages trigger photoreceptor cell death leading to blindness ${ }^{2,3}$. With its functional tight 50 junctions, the RPE monolayer and the choriocapillaris, separated by a proteinaceous (2-5 $\mu \mathrm{m}$ thick) Bruch's 51 membrane, form the outer-blood-retina-barrier (oBRB) in the back of the eye ${ }^{4}$. RPE basal infoldings,

52 Bruch's membrane, and fenestrations (60-80 nm pores) in the endothelial cell (EC) membrane allow 53 unimpeded macromolecule and nutrient flow from the blood into the RPE - which regulates flow to the 54 photoreceptors ${ }^{4}$.

55 Dry AMD initiates by the accumulation of lipid/protein-rich drusen deposits, triggered by complement 56 pathway activation, in the subRPE region ${ }^{3,4}$. Disease progression to the advanced stage geographic atrophy 57 is hallmarked by RPE dropout that precedes choriocapillaris degeneration, leading to outer retina starvation ${ }^{3}$. 58 In contrast, in advanced wet AMD (choroidal neovascularization, CNV) choriocapillaris hyperproliferate, 59 grow under the RPE and occasionally break through RPE tight junctions leaking blood in the sub-retinal 60 space ${ }^{5}$. This separates the photoreceptors from the RPE and deprives them of the RPE's functional and 61 nutrient support resulting in photoreceptor degeneration ${ }^{5}$. The importance of vascular endothelial growth 62 factor (VEGF) in CNV is underscored by the successful application of drugs that block VEGF signaling 63 (Bevacizumab, Ranibizumab, and Aflibercept $)^{6,7}$. However, the mechanism of increased VEGF secretion 64 by human RPE remains elusive due to a lack of human-relevant models that accurately recapitulate CNV. 65 Here, we developed a functionally validated 3D-oBRB utilizing bioprinting, tissue engineering, and 66 directed differentiation of human induced pluripotent stem cells (iPSCs). The 3D-oBRB model recapitulates

67 RPE-choriocapillaris interactions under healthy and dry and wet AMD stages. 


\section{Design of 3D outer blood-retina barrier (3D-oBRB)}

72 To develop a functional 3D-oBRB, we included the four key cell types (RPE, ECs, pericytes, and fibroblasts)

73 in our tissue design (Fig. 1a) ${ }^{8,9}$. The identity of iPSC-derived ECs (iECs), iPSC-derived RPE (iRPE),

74 primary ECs, pericytes, and fibroblasts was confirmed using cell type-specific markers (CD31, ETV2, vWF

75 - ECs; NG2, PDGFR- $\beta$, COL-I - pericytes; VIMENTIN, COL-I, PDGFR- 3 (negative) - fibroblasts; MITF,

76 TYRP1, ZO-1, RPE65, EZRIN - RPE, Fig. S1) ${ }^{10-12}$. A biodegradable scaffold made of thermally fused

77 electrospun poly-(lactic-co-glycolic acid) (PLGA) fibers of 400-500 nm diameter supported the formation

78 of a polarized RPE monolayer on one side and a capillary-bed derived from 3D-printed bioink of ECs,

79 pericytes, and fibroblasts on the other side (Figs. 1a and S2a,b). As the tissue matured, the degrading

80 scaffold was gradually replaced by extracellular matrix (ECM) secreted by RPE and ECs forming a Bruch's

81 membrane-like structure between the RPE and the capillary-bed.

82 Manufacturing and functional maturation of the 3D-oBRB took 42 days (Fig. 1a). The printing surface was

83 prepared a day before bioprinting by replacing the snapwell membrane with a $12 \mathrm{~mm}$ PLGA scaffold disc

84 (Fig. 1a; methods). The scaffold's RPE side was coated with vitronectin $(50 \mu \mathrm{g} / \mathrm{mL})$ to aid cell attachment.

85 To enhance scaffold hydrophilicity - needed for bioink attachment, scaffold was treated with oxygen plasma

86 (5cc/min, 30mins). Improved hydrophilicity was confirmed by the dispersal of a water droplet (Figs. 1a, 87 S2c, d). 
Song et al. Figure 1

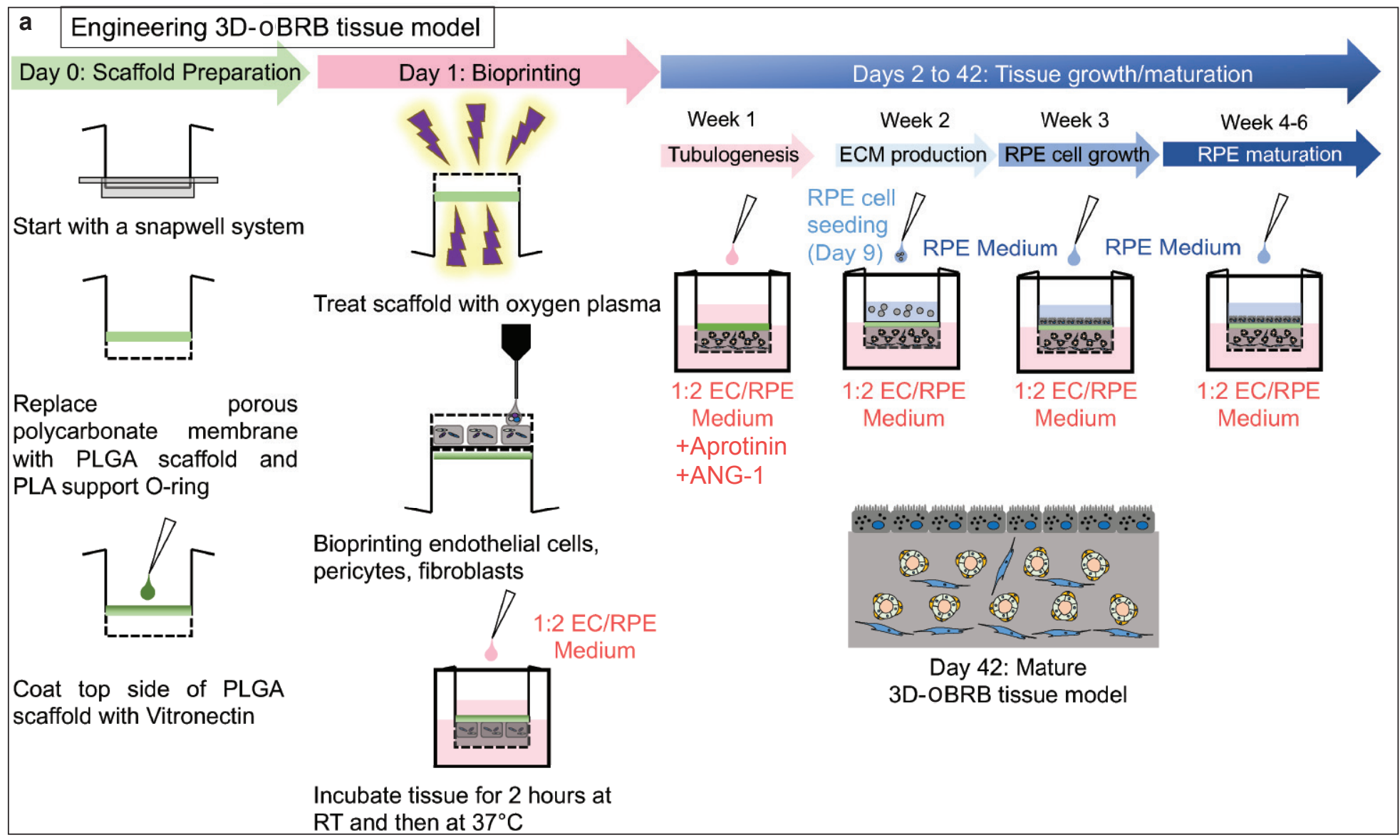

$\mathrm{RT}$ and then at $37^{\circ} \mathrm{C}$
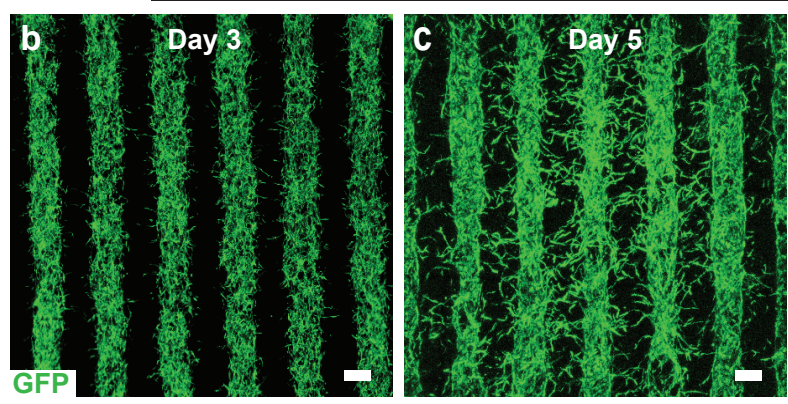

Day 9
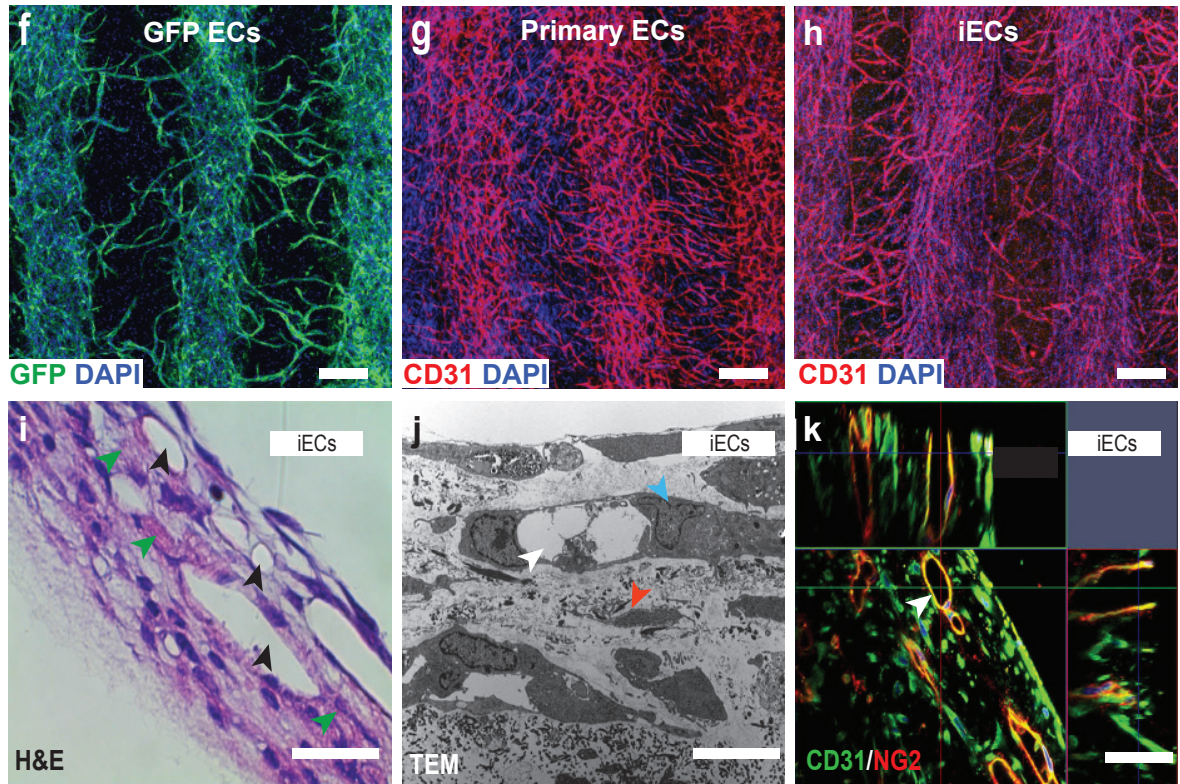

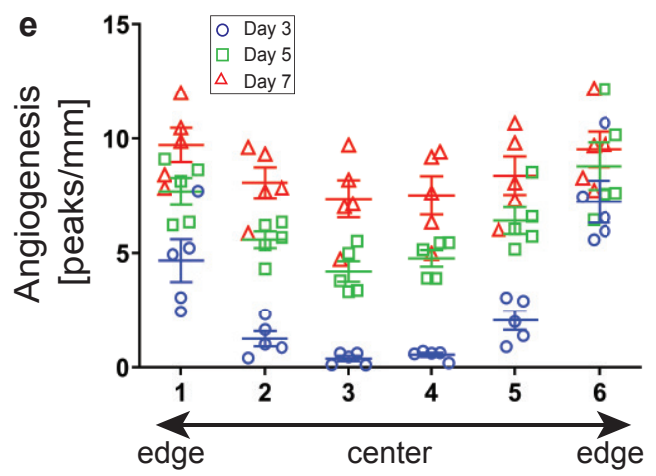


93 Fig. 1 | Design of 3D-oBRB. a, Bioprinting workflow with human endothelial cells (ECs) and RPE. b-d,

94 Vascular development of GFP expressing primary ECs on day 3 (a), day 5 (b) and day 7 (c) after printing.

95 Scale bars, $500 \mu \mathrm{m}$. e, Angiogenesis between printed stripes $(\mathrm{n}=5)$. $\# \mathrm{p}<0.05$ in day 3 vs. day 5 , $\uparrow \mathrm{p}<0.05$ in 96 day 5 vs. day $7,{ }^{*} \mathrm{p}<0.05$ in day 3 vs. day 7. f-h, Vascular formation of GFP-positive ECs (green, f), primary

97 ECs (CD31 - red, g), and iPSC-derived ECs (iECs, CD31 - red, h) and nuclei (blue). Scale bars, $500 \mu \mathrm{m}$.

98 i, H\&E images of $10 \mu \mathrm{m}$ thick cross section of iECs derived vascular tissue (day 7). Vasculature is marked

99 with black arrowheads, and ECM components are marked with green arrowheads. Scale bar, $50 \mu \mathrm{m} . \mathbf{j}$,

100 Transmission electron microscope (TEM) images of iECs derived vascular tissue at day 7 . White arrowhead

101 shows a capillary, blue arrowhead marks pericytes, and red arrowhead labels fibroblasts. Scale bar, $300 \mathrm{~nm}$.

$102 \mathbf{k}$, Orthogonal views of confocal images of $100 \mu \mathrm{m}$ thick tissue sections stained with CD31 (ECs; green)

103 and NG2 (pericytes; red). White arrowheads mark patent EC-derived capillaries. Scale bar, $50 \mu \mathrm{m}$.

104 Statistical significance was attributed to values of $p<0.05$ as determined by two-way ANOVA and

105 Bonferroni post-hoc pair comparison. All error bars indicate STE.

106

107

108

109

110

111

112

113

114 
116 To produce a dense capillary-bed of $5-20 \mu \mathrm{m}$ lumen diameter ${ }^{13}$, we sought to bioprint a high density of 117 mixed ECs, pericytes and fibroblasts (12/6/0.6 million fibroblasts/EC's/pericytes per mL of bioink). To 118 achieve a homogenous bioink with such high cell density, we designed a temperature-sensitive hydrogel by 119 mixing a gelatin-based hydrogel (Novogel, $60 \mathrm{mg} / \mathrm{mL}$ ) with fibrinogen $\left(2.5 \mathrm{mg} / \mathrm{mL}\right.$ ) at $37^{\circ} \mathrm{C}$ reduced 120 hydrogel viscosity allowing bioink homogeneity and easier loading in the printing syringe. Cooling the 121 syringe down to $10^{\circ} \mathrm{C}$ increased bioink viscosity allowing for easy printing of desired structures. During 122 tissue culture at $37^{\circ} \mathrm{C}$, Novogel dissolved and fibrin continued to provide the $3 \mathrm{D}$ architecture needed to 123 support capillary-bed formation. However, fibrin degraded after four days leading to capillary-bed collapse, 124 as confirmed by the clumping of GFP-expressing ECs (Figs. S3a,b). The addition of recombinant aprotinin $125(25 \mu \mathrm{g} / \mathrm{mL})$, a known fibrinolysis inhibitor, prevented tissue collapse giving fibroblasts time to secrete ECM 126 that enabled a stable capillary network (Figs. S3c,d).

128 A striped bioprinting pattern facilitated capillary growth quantification outwards from the printed stripes 129 into the acellular hydrogel (movie S1; Fig. S4). A MATLAB-based algorithm was developed for angiogenesis quantification (Methods). Bioprinted tissue was treated with VEGF $(85 \mathrm{ng} / \mathrm{mL})$ for three or 131 seven days, to determine the optimal treatment window for capillary growth. Capillary sprouting was 132 evident in printed ECs with three-days of VEGF treatment, but these sprouts did not form a contiguous 133 capillary-network and disintegrated by day 7 (arrowheads, Fig. S4a). In comparison, seven-days treatment 134 increased capillary angiogenesis resulting in anastomosis between two printed stripes (arrowheads, Fig. 135 S4b, movie S1). Quantification revealed 1.5-2x folder higher angiogenesis on the edges of the printed 136 tissues and 5-10x fold more angiogenesis in the center of the acellular structure after seven-days VEGF 137 treatment as compared to three-days treatment (Figs. S4a-e), establishing the use of exogenous VEGF for 138 seven days. 
Exogenous VEGF treatment supported EC angiogenesis, but it also increased EC migration resulting in 140 sole ECs that didn't incorporate into capillaries ${ }^{14}$ (Figs. S5a and b - circle). To prevent this undesirable EC 141 migration, we supplemented tissues with recombinant angiopoietin-1 (ANG-1; $100 \mathrm{ng} / \mathrm{mL}$ ), a well-known 142 EC migration inhibitor. Expectedly, ANG-1 initially slowed down capillary sprouting (arrowheads Figs.

143 S5a and c), but it did not disrupt capillary formation. In fact, by day 8 the number of capillaries increased 144 in ANG-1 treated Group 2 as compared to without it (Group 1) (Figs. S5 b,d). This allowed more precise 145 analysis of the angiogenesis kinetics excluding individual EC signal.

To monitor the time course of angiogenesis, we analyzed the GFP-expressed ECs on days 3,5 and 7 postprinting. By day 3, capillaries grew in a gradient with 5-6 capillary peaks/mm close to the printed stripe edge the and no peaks in the center of the acellular zone ( $p<0.001$; Figs. 1b, e, S6a). By day 5, the capillary gradient between stripe edges and the acellular area center was shallowed with 8 peaks $/ \mathrm{mm}$ on edges and 4 peaks $/ \mathrm{mm}$ in the center ( $<<0.05$; Figs. $1 \mathrm{c}, \mathrm{e}, \mathrm{S} 6 \mathrm{~b})$. By day 7 , no statistically significant difference between edges $(9$ peaks $/ \mathrm{mm})$ and center $(10$ peaks $/ \mathrm{mm})$ was evident $(\mathrm{p}>0.05$; Figs. 1d, e, S6c). A comparative analysis of capillary networks derived from GFP-expressing ECs, non-GFP expressing ECs, and iECs 154 revealed similar capillary confluency and angiogenesis throughout the tissue (Figs. 1f-h). Together, this 155 confirmed robustness of our bioprinting protocol across different ECs. Histological analysis (H\&E staining)

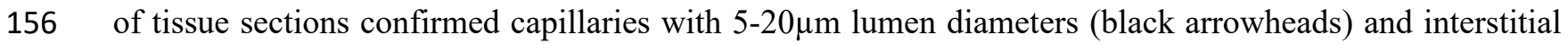
157 spaces filled with cells and ECM (green arrowheads; Fig. 1i). Transmission electron microscopy (TEM) of tissue cross-sections confirmed iECs derived capillaries (white arrowhead) with a pericyte (blue arrowhead) wrapped around, and fibroblasts (red arrowhead) in interstitial spaces (Fig. 1j). This observation was further confirmed in confocal images of $100 \mu \mathrm{m}$ thick tissue slices where pericytes (NG2, red) were found to colocalize with iEC (CD31, green) derived intact capillaries (arrowhead; Fig. 1k, movie S2). Overall, this

162 data shows that our bioink composed of Novogel, fibrinogen mixed with fibroblasts, ECs, and pericytes 163 (2:1:0.1 ratio) forms a robust capillary-network with both primary and iPSC-ECs. 
165 To complete the 3D-oBRB, iPSC-derived RPE cells were seeded and matured $^{12}$ on the Vitronectin-coated 166 scaffold side. TEM confirmed iRPE cells-initiated polarization and pigmentation within three weeks after 167 seeding (Fig. 2a). By six weeks, known structural features of RPE maturation and polarization were evident: 168 dense apical processes (ap), tight junctions ( $\mathrm{tj}$ ) between neighboring cells, apically located stage IV 169 melanosomes (ml), and basal infoldings (bi) - a critical native-RPE feature that cannot be reproduced in 170 RPE grown on plastic substrates ${ }^{15}$ (Fig. 2b). Similar to the native-BRB, RPE basal infoldings were in 171 continuum with the ECM that had replaced the scaffold and formed a Bruch's membrane (BM)-like 172 structure (Fig. 2b) ${ }^{16}$. Immunostaining for the tight junction marker E-CADHERIN and the apical process 173 marker EZRIN further confirmed RPE monolayer junctional maturity and polarization (Fig. 2c). Concurrent 174 with the RPE maturation, capillary confluency was evident in CD31 immunostained 3D rendered tissue 175 images (Fig. 2d). High expression of FELS, a fenestration marker ${ }^{17}$, colocalized with CD31 suggested the 176 formation of fenestration in our 3D-oBRB model (Fig. 2e, movie S3). Histological analysis revealed the 177 structure of the entire 3D-oBRB with a 2-4 $\mu \mathrm{m}$ thick BM-like acellular ECM region sandwiched between 178 an RPE monolayer on top and a 40-50 $\mu \mathrm{m}$ thick vascular tissue with capillaries (cl) running along various 179 tissue planes (Fig. 2f). To confirm functionality of the 3D-oBRB tight junctions, we measured tissue 180 resistance to current flow (trans-epithelial resistance - TER), produced by functional tight junctions between 181 neighboring RPE cells. TER of the tissue with vasculature and no iRPE layer was $53.4( \pm 1.36) \mathrm{Ohms} \cdot \mathrm{cm}^{2}$. 182 The presence of the iRPE monolayer increased the tissue TER to $740.4( \pm 155.99) \mathrm{Ohms} \cdot \mathrm{cm}^{2}$, comparable 183 to the TER of iRPE monolayer without the vascularized tissue 873.7 ( \pm 67.82$)$ Ohms.cm² (Fig. 2g). Finally, 184 to confirm capillaries' functionality in this $3 \mathrm{D}-\mathrm{oBRB}$, we transplanted the intact tissue in the choroid of 185 immunocompromised rats. Two weeks post-transplantation, animals were perfused with DiI in the left heart 186 ventricle to label all the vessels; and choroid was analyzed histologically. Anti-human STEM121 labeling 187 with GFP-expressed ECs in $10 \mu \mathrm{m}$ cryosections identified human capillaries perfused with DiI suggesting 188 anastomosis and integration with rat capillaries (Fig. 2h). This was further confirmed by $100 \mu \mathrm{m}$ sections 
189 that revealed different size human capillaries anastomosed and integrated within rat capillaries (Figs. 2i, 190 S7a, b). Overall, TEM, immunostaining, barrier resistance, and transplantation confirmed the formation of 191 a functional 3D-oBRB with polarized RPE monolayer and functionally lumenized capillaries.

192

193

194

195

196

197

198

199

200

201

202

203

204

205

206

207

208 


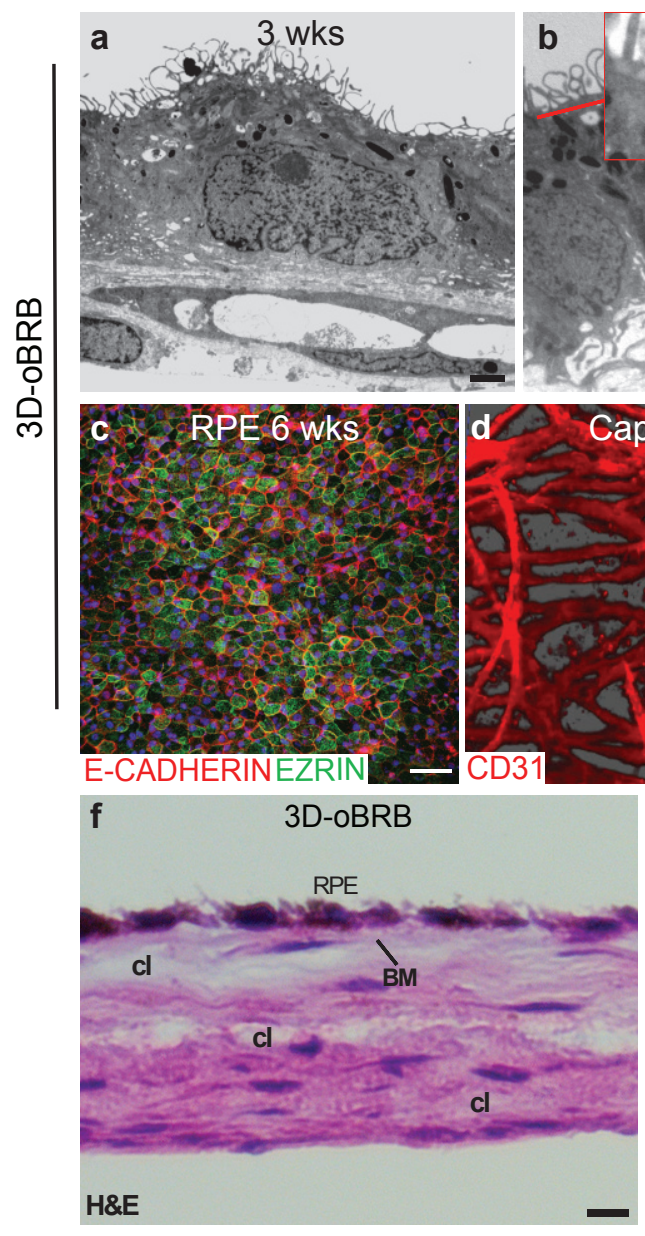

$$
\text { MCD } 6 \text { wks ap }
$$

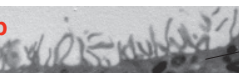


Fig. 2 | Engineering of 3D oBRB. a,b, TEM images of 3D-oBRB maturity at week 3 (a) and 6 (b) with

210 RPE pigmentation: melanin (ml), RPE apical processes (ap), RPE basal infoldings (bl), tight junction (tj)

211 formation and Bruch's membrane (BM). Scale bars, $1 \mu \mathrm{m}(\mathbf{a}), 2 \mu \mathrm{m}(\mathbf{b})(\mathrm{n}=3) \mathbf{c}$, Immunostaining for RPE

212 maturity markers E-CADHERIN (red) and EZRIN (green) in 6-week-old tissues. Scale bar, $50 \mu \mathrm{m}$. d, 3D-

213 rendered image of CD31 (red) immunostained capillary-bed in 6-week-old 3D-oBRB. Scale bar, 50 $\mu$.

$214(\mathrm{n}=4)$ e, Immunostaining for capillary-maturation marker FELS (red) co-labled with EC marker CD31

215 (green) of confocal images of vascular networks labeled with CD31 (red). Scale bar, 50 $\mu$ m. (n=3) f, H\&E

216 staining of 6-week-old tissues containing capillaries (cl), RPE, and Bruch's membrane (BM). Scale bar, 10

$217 \mu \mathrm{m} . \mathbf{g}$, Transepithelial resistance (TER) of 3D-oBRB compared to vascular and 2D-RPE (n=3). $\mathbf{h}$, GFP

218 signal in human ECs and immunostaining with STEM121 (magenta) detect human capillaries that are 219 perfused with DiI (yellow) in $10 \mu \mathrm{m}$ cryosections of rat choroid transplanted with 3D-oBRB. Scale bar 75

$220 \mu \mathrm{m}$. ( $\mathrm{n}=8$ eyes from 4 animals). i, Immunostaining for STEM121 (magenta) detects human capillaries

221 integrated with rat capillaries detected by Dil (yellow) perfusion in $100 \mu \mathrm{m}$ vibratome sections of

222 transplanted rat choroid. Scale bar $100 \mu \mathrm{m}$. Statistical significance was attributed to values of $\mathrm{p}<0.05$ as

223 determined by one-way ANOVA with Tukey's multiple-comparisons test. ${ }^{* * *} \mathrm{p}<0.0001$, All error bars

224 indicate STE.

225

226

227

228

229

230 


\section{RPE cells induce capillary fenestration in 3D-oBRB}

233 Strong FELS expression in fully-mature 3D-oBRB (Fig. 2e) suggested the presence of fenestration in 234 capillaries. To better characterize the fenestration process, we performed a temporal analysis of FELS 235 expression in 3D-oBRB constructs (Figs. S8a-d). CD31 co-immunostaining revealed minimal FELS 236 expression in capillaries for the first two weeks post-printing (Figs. S8a, b). Coincidental with iRPE 237 monolayer polarization (Fig. 2a), FELS expression became prominent starting week three (Figs. S8c, d). 238 To confirm if FELS expression led to fenestration in fully-mature 3D-oBRB, we performed ultrastructural analysis of capillaries (Figs. 3a, b). TEM revealed 50-80 nm thinned areas in the EC capillary membrane reminiscent of fenestration in native choroidal capillaries (arrowheads Fig. 3b) ${ }^{18}$. To further confirm if FELS expression was affected by the RPE presence, we cultured the vascular tissue with (Figs. 3c, d, g) or without the RPE (Figs. 3e, f, g). CD31 and FELS co-immunostaining revealed a confluent capillary bed with higher capillary number and thickness, and a 2.5-fold higher FELS expression in tissues that contained the RPE as compared to tissues that lacked the RPE monolayer where vasculature collapsed (Figs. 3d, f, g). Overall, this work provides the first direct evidence that human RPE cells induce fenestration in an iECderived capillary-network.

RPE-dependent fenestration formation in our 3D-oBRB suggested that iECs acquire a choroidal fate. To investigate this possibility, we compared the transcriptome profiles of iECs within the 3D-oBRB and 2D monocultures (without RPE) using scRNAseq. Clustering samples by t-distributed stochastic neighbor embedding (tSNE) plots revealed three different EC populations in the 3D-oBRB, likely based on location

252 in the tissue: fully-mature (FM) ECs expressed several choroidal and arterial maturation genes; partially253 mature (PM) ECs expressed fewer arterial maturation genes; and inflamed (Inf) ECs expressed 254 inflammatory genes in addition to arterial maturation genes (Figs. 3h,i, Table S3). Detailed analysis 255 revealed genes relevant for ECM (COL3A1, COL6A1, COL1A1), EC maturation (LOX, VEGFA, GJA4, $256 A C E$ ), choroidal phenotype (APOE, PLVAP, TIMP1), angiogenesis (downregulated - ENG, ACTB, MMP1; 
257 upregulated - ADAM17, ITGAV), and arterial maturation (EPSA1, KDR, EFNB2, and NOTCH1) showed 258 the most differential expression among the 531 genes that were significantly different between 2D and 3D 259 iEC transcriptomes (Figs. 3i, S9, S10) ${ }^{19-23}$. Overall, these findings suggest that our 3D-oBRB construct 260 capillaries matured and attained an arterial and choroidal phenotype.

261

262

263

264

265

266

267

268

269

270

271

272

273

274

275

276 


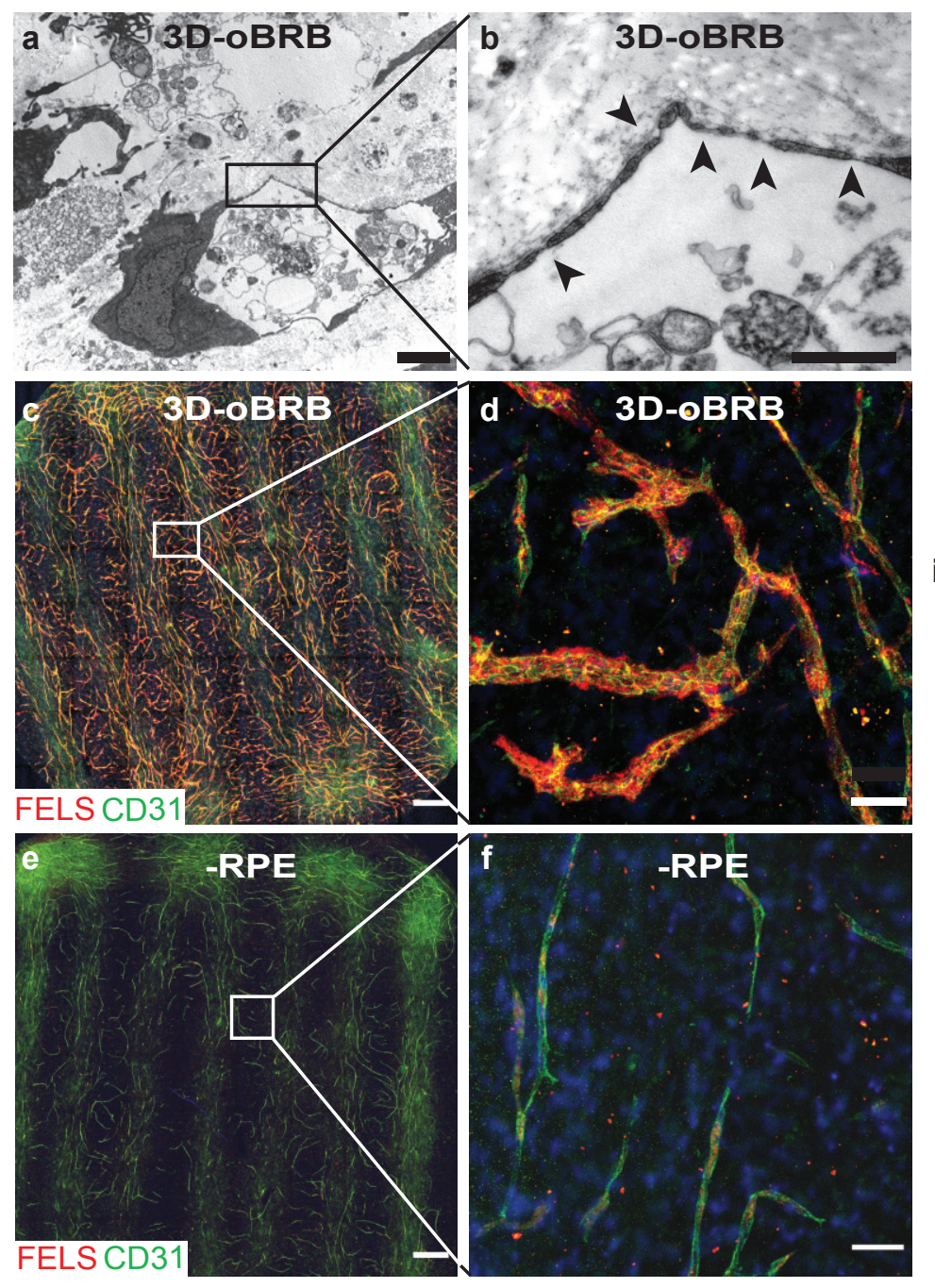

g

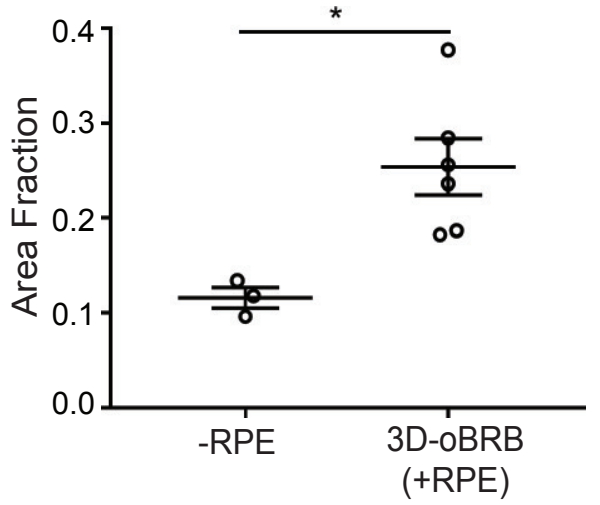

h

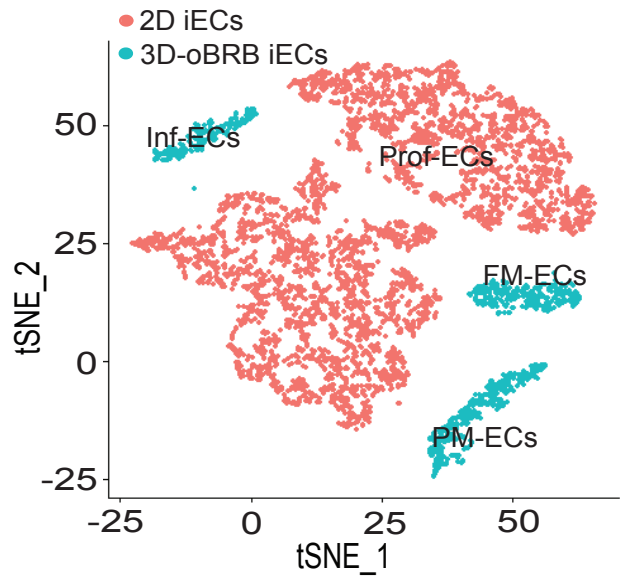

i

2D iECs

iECs (3D-OBRB)

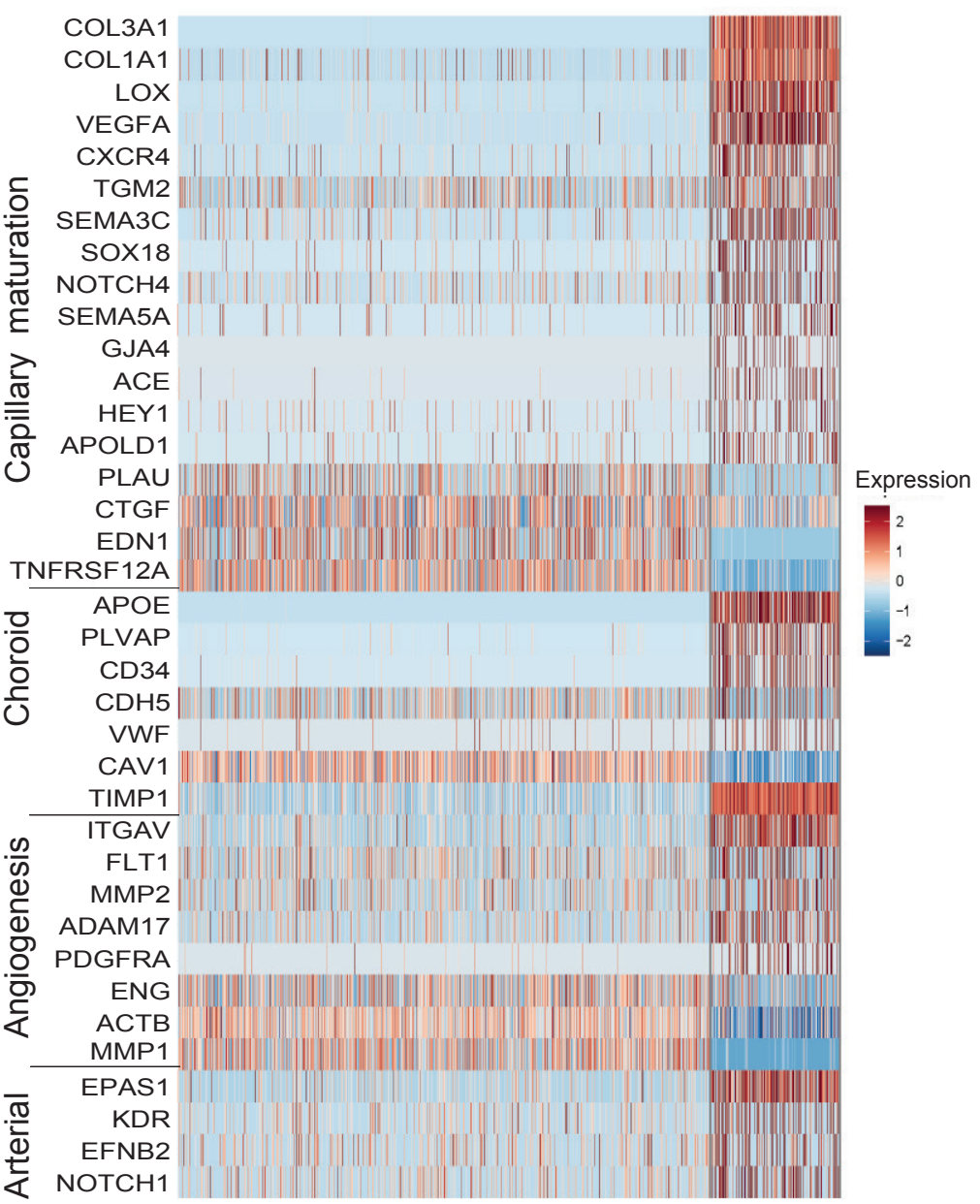


277 Fig. 3 | Capillary maturation in 3D-oBRB. a,b, TEM images highlight fenestration (arrowheads) in iECs 278 with in 3D-oBRB capillaries at week 6. Scale bars, $2 \mu \mathrm{m}(\mathbf{a}), 500 \mathrm{~nm}(\mathbf{b}) .(\mathrm{n}=3)$. c-f, Immunostaining for 279 FELS (red) and CD31 (green) in 3D-oBRB (with RPE; c, d) or just the vasculature (without RPE; e, f). 280 Scale bars, $500 \mu \mathrm{m}(\mathbf{c}, \mathbf{e})$ and $50 \mu \mathrm{m}(\mathbf{d}, \mathbf{f})$. g, Area fraction of FELS and CD31 expression in vascular 281 regions with or without RPE. ( $\mathrm{n}=3$ ). $\mathbf{h}$, TSNE plots from sc-RNA seq of 2D iECs and iECs from 3D-oBRB. $282 \mathrm{i}$, Gene expression differences between 2D iECs and iECs from 3D-oBRB for genes related to endothelial 283 maturation, choroid, angiogenesis, and arterial development. Statistical significance was attributed to 284 values of $\mathrm{P}<0.05$ as determined by unpaired t-test. Data depicts results from $\mathrm{n}=3012$ cells $(2 \mathrm{D} R \mathrm{RE}), \mathrm{n}=$ 2854380 cells (3D-oBRB RPE), $\mathrm{n}=5369$ cells $(2 \mathrm{D} \mathrm{iECs})$, and $\mathrm{n}=1294$ cells $(3 \mathrm{D}-\mathrm{OBRB} \mathrm{iECs}) .{ }^{*} \mathrm{p}<0.05$, All 286 error bars indicate STE.

287 288 289 290 291 292 293 294 295 296 


\section{Bruch's membrane formation in 3D-oBRB}

TEM suggested the formation of a Bruch's membrane-like structure between the RPE and the capillarybed (Fig. 2b). We confirmed strong immunostaining for ECM components of native Bruch's membrane including FIBRONECTIN, LAMININ, COLLAGEN IV (COL IV), and ELASTIN ${ }^{16,24}$ (Figs. 4a-d, movie S4). 3D rendering of ELASTIN and LAMININ immunostained images further revealed an acellular membranous zone underneath the RPE monolayer (movie S4). Quantification revealed a 15x higher COL IV expression and 4x higher ELASTIN expression in 3D-oBRB as compared to 2D-iRPE (Figs. 4 e-i). To determine the cellular origin of Bruch's membrane, we analyzed ECM-related gene expression in scRNAseq data. iRPE contributed to ECM proteins like COL8AI and EFEMPI - involved in Doyne honeycomb retinal dystrophy ${ }^{25}$ whereas iECs were the major source of ECM proteins including COL8A1, COL8A2, COL9A3, COL11A1, ELN (Figs. S10a, b) ${ }^{26-28}$. All together this data shows that our 3D model enabled the creation of a Bruch's membrane-like structure in vitro that further enabled close interactions between RPE and choriocapillaris.

The presence of a Bruch's membrane-like structure prompted the question if iRPE grown on a natural ECM behave differently than iRPE grown on plastic substrates ${ }^{15}$. scRNAseq data confirmed this hypothesis. 46 out of the previously reported 157 RPE signature-genes ${ }^{29}$ were $1.5-4 \log 2$ fold-higher in iRPE in the 3DoBRB than 2D iRPE. Most notable were components of the visual cycle (RPE65, RDH5, RBP1, RLBP1, RDH11), angiogenic and non-angiogenic genes (VEGFA, ENPP2 and SERPINF1), components of the Bruch's membrane (EFEMP1, ITGAV, ITM2b and TIMP1), and exosome assembly genes (HSP90B1, LAMP2, SDCBP) (Figs. 4j, k, S11a, b). Overall, this data provides additional evidence that as part of the 3D-oBRB both iECs and iRPE attain more native-like properties including the formation of a Bruch's membrane equivalent structure. 

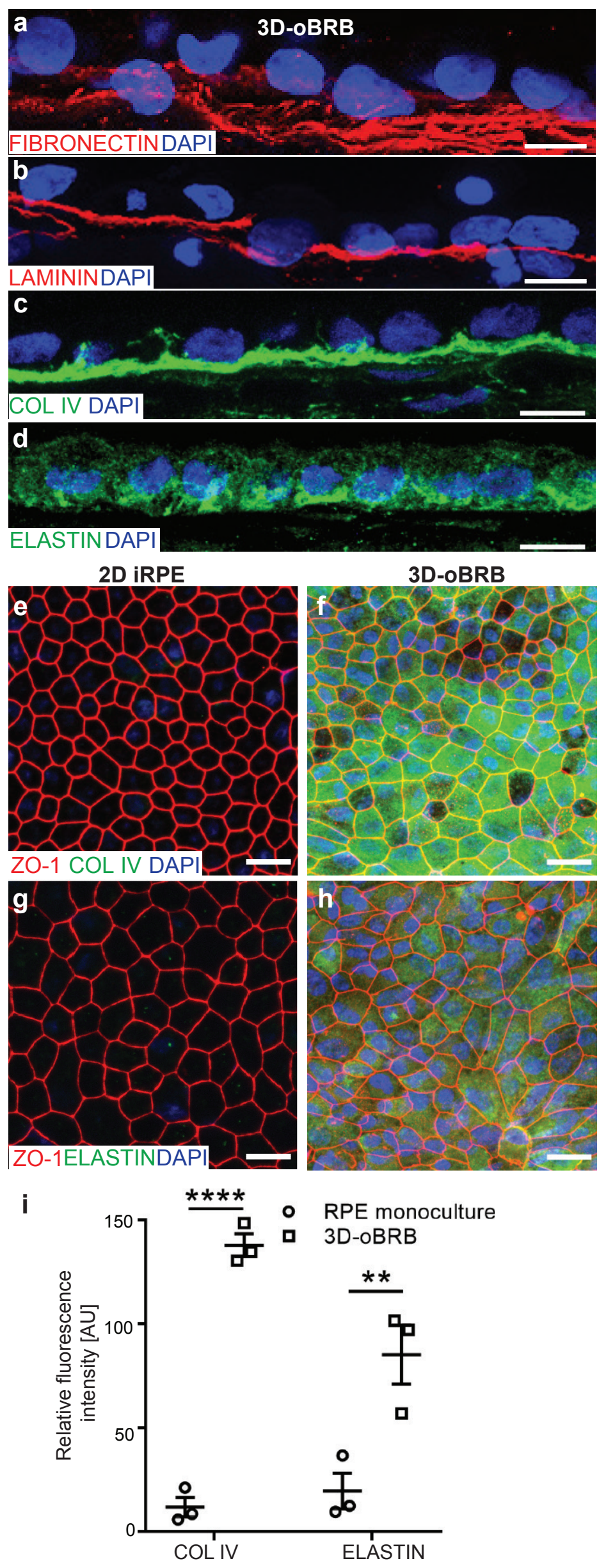

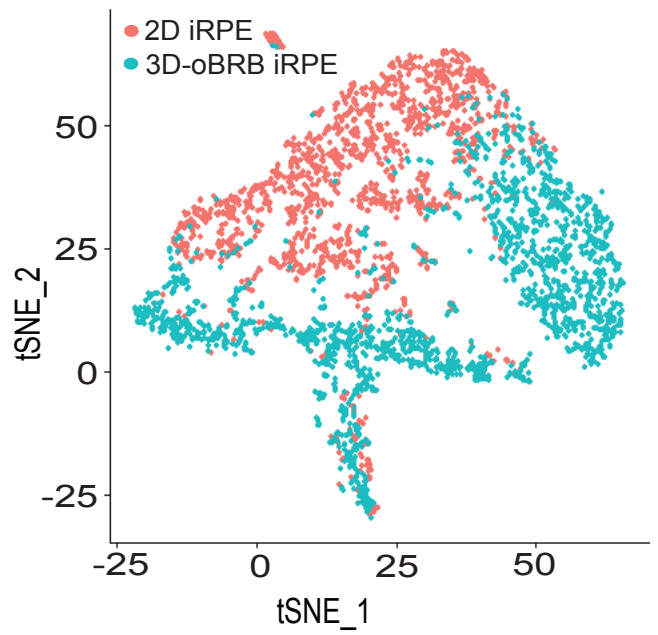

2D iRPE iRPE (3D-oBRB)

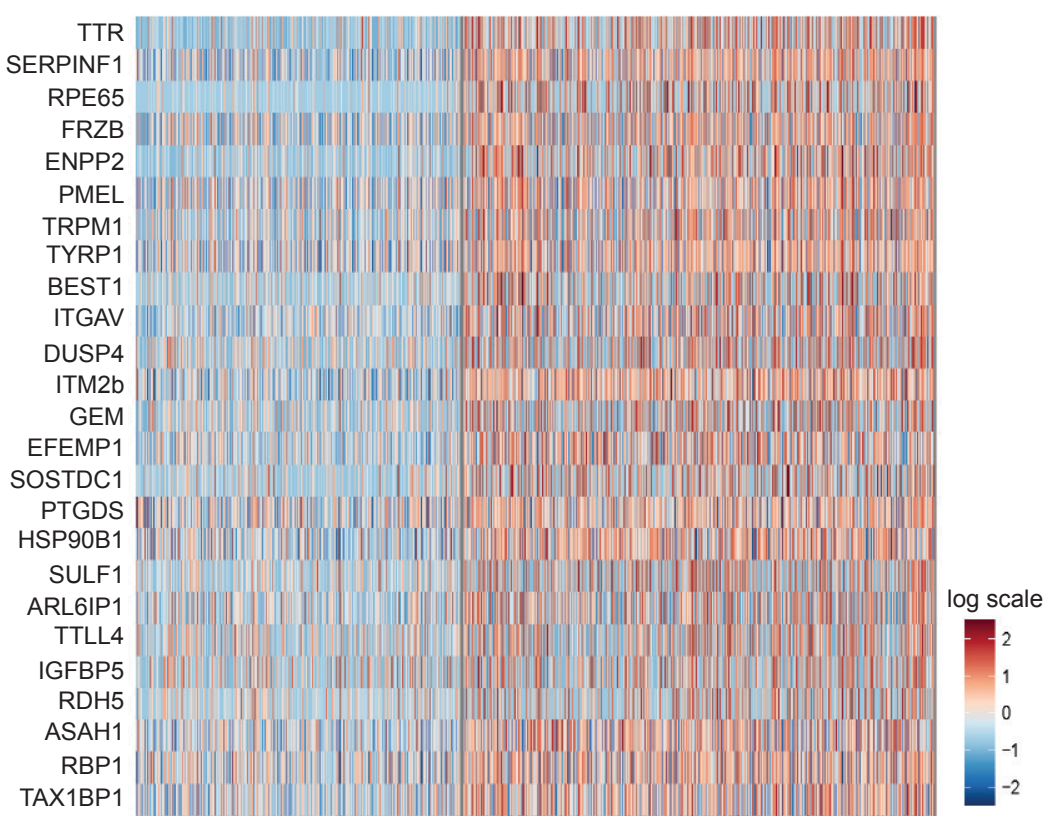


323 Fig. 4 | RPE maturity in 3D-oBRB. a-d, Cross sections of 3D-oBRB immunostained for Bruch's 324 membrane proteins FIBRONECTIN (a), LAMININ (b), COLLAGEN IV (COL IV) (c), and ELASTIN (d). 325 Nuclei stained with DAPI. Scale bars, $10 \mu \mathrm{m}$. e-h, 2D RPE monoculture (e, g) and 3D-oBRB (f, h), co326 immunostained for COL IV (green) or ELASTIN with ZO-1 (red), and nuclei (blue). Scale bars, $30 \mu \mathrm{m}$. i, 327 Fluorescence mean intensity comparison of ELASTIN and COL IV immunostaining in 2D-iRPE and 3D328 oBRB models. $(n=3)$. $\mathbf{j}$, tSNE plots from sc-RNA seq of 2D-iRPE and RPE in 3D-oBRB. k, Gene 329 expression of RPE signature genes, comparison between 2D-iRPE and RPE in 3D-oBRB. Statistical 330 significance was attributed to values of $\mathrm{p}<0.05$ as determined by two-way ANOVA and Sidak's multiple 331 comparison test. $* * \mathrm{p}<0.01, * * * * \mathrm{p}<0.0001$. All error bars indicate STE.

332 


\section{D-oBRB recapitulates choroidal phenotypes seen in dry and wet AMD}

346 Drusen - a hallmark of dry AMD - accumulates under the RPE within the Bruch's membrane ${ }^{30}$. Complement 347 competent human serum (CC-HS), has recently been linked to drusen formation ${ }^{31}$. Treatment of 3D-oBRB with 5\% CC-HS induced APOE positive drusen-like deposits within the Bruch's membrane, as confirmed by histological analysis of 3D-oBRB in en-face view (APOE - purple, Figs. 5a,d), cross-sections (white lines, APOE - purple, Figs. 5g), and 3D rendered images (APOE - purple, movie S5). CC-HS treatment also led to RPE atrophy in 3D-oBRB (compare F-ACTIN - green Figs. 5b, e). Consistent with RPE atrophy, capillary degeneration was evident with CC-HS treatment (arrowheads; CD31 - red, Figs. 5c, f and g). Lastly, these structural changes in the 3D-oBRB led to a loss in its barrier resistance, as confirmed by a 20x drop in TER ( $p<0.01$; Fig. 5 h). Overall, these results validated 3D-oBRB as a physiologically relevant dry AMD model including subRPE drusen deposits, loss of barrier resistance, RPE atrophy, and capillary degeneration.

It is thought that hypoxia in the back of the eye leads to stabilization and nuclear translocation of the transcription factor HIF- $1 \alpha$ in the RPE ${ }^{32}$. HIF- $1 \alpha$ increases the expression and secretion of VEGF ${ }^{33}$, which leads to wet AMD. However, there is no direct proof to support this hypothesis for human ocular tissues. We sought to recreate wet AMD using RPE-specific hypoxia in the 3D-oBRB model. Treatment of the iRPE apical side with ML228 $(2 \mu \mathrm{M})$, a known HIF-1 $\alpha$ activator $^{34}$, led to HIF-1 $\alpha$ activation in $<5 \%$ of cells by 48 hours; by 96 hours elevated HIF-1 $\alpha$ protein levels was evident in $25 \%$ of cells (Figs. S12a, b, d, e).

364 Continued treatment of ML228 for two weeks resulted in HIF-1 $\alpha$ activation in majority of iRPE cells and 
370 Treatment of mature 3D-oBRB (on the apical side) with ML228 produced outcomes similar to seen in 2D-

371 iRPE, including disruption of tight junctions and a 3-fold drop in tissue TER (Figs. 5i, j, o). Unlike 2D

372 iRPE, in the 3D-oBRB model the TER did not drop to zero suggesting a protective effect of the capillary-

373 bed on iRPE barrier resistance (Figs. S12g, 5o). Activation of HIF-1 $\alpha$ in the RPE of a mature 3D-oBRB

374 initiated a CNV-like response with capillaries hyperproliferating towards the iRPE monolayer, as confirmed

375 by image-based analysis of z-planes (Figs. 5k, 1; yellow to red color of the code shows capillaries that are

376 in the subRPE zone - white circles). Cross-sections of 3D rendered images of tissues clearly revealed

377 capillaries hyperproliferating into the Bruch's membrane and expanding into the subRPE zone (Figs. 5 m,

$378 \mathrm{n}$ - arrowheads; movie S6). To determine if the CNV-like response seen in our hypoxic 3D-oBRB model

379 was VEGF induced, we measured VEGF secretion in ML228 treated 3D-OBRB. ML228 treatment led to 380 a 5-fold increase in the apical VEGF secretion (Fig. 5p), while basal VEGF secretion did not change with 381 ML228 treatment (Fig. 5q). Quantification of VEGF immunostaining revealed that most of the basally 382 secreted VEGF accumulated around capillaries, likely binding to its cognate receptor - leading to CNV-like 383 phenotype (Fig. 5r). Overall, the above data confirmed that our 3D-oBRB is able to recapitulate both dry 384 and wet AMD phenotypes in vitro. 


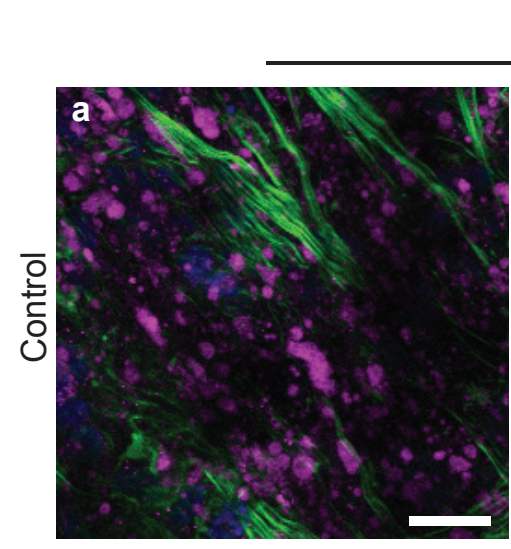

APOEF-ACTINCD31DAPI
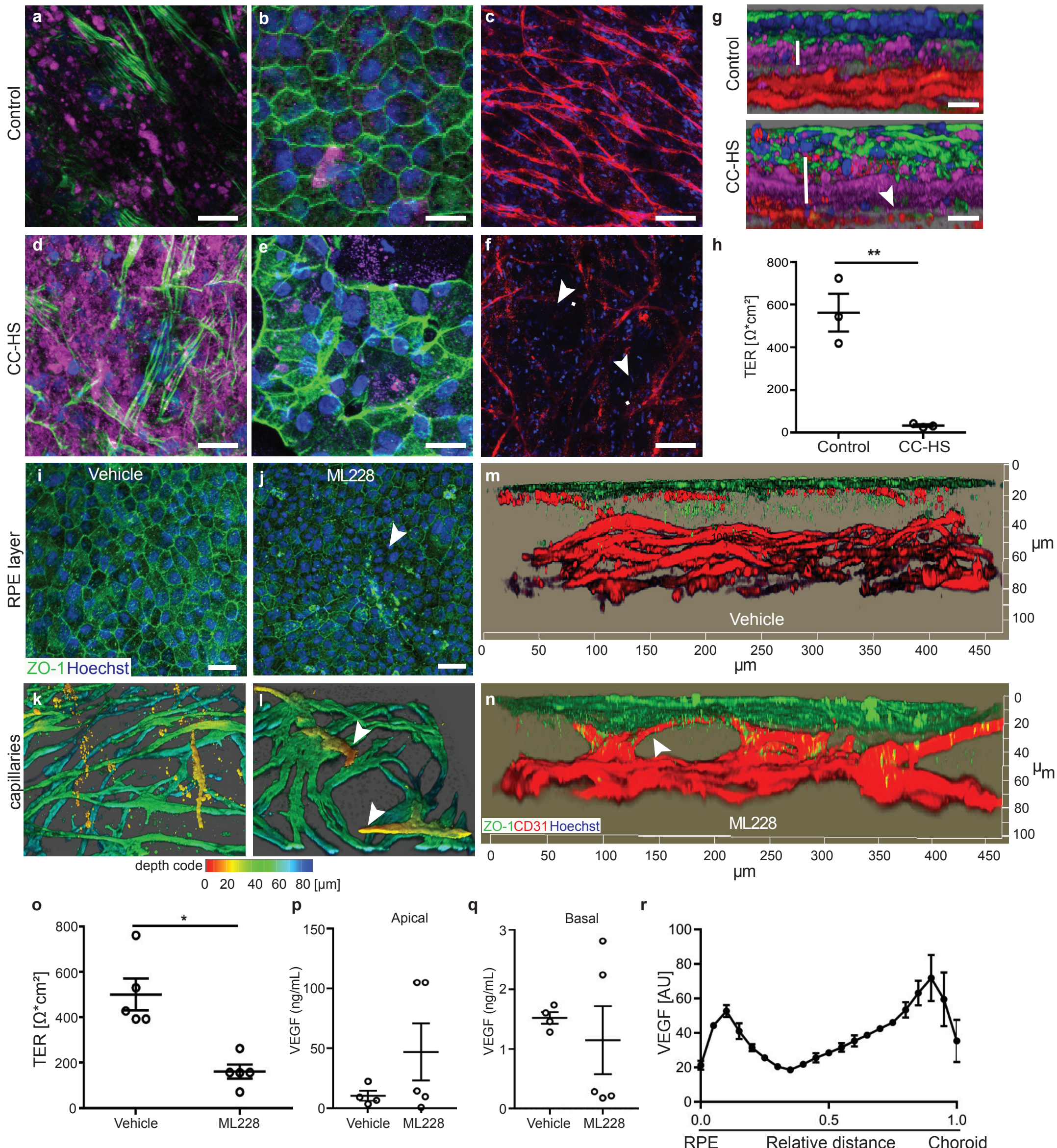

(a-f), cross-section (g) views of APOE (magenta), CD31 (red) immunostaining, F-ACTIN (green), Hoechst (blue) in complement competent human serum (CC-HS) treated and control 3D-oBRB. Arrowheads mark degenerated RPE and capillaries. Scale bars, $100 \mu \mathrm{m}$. (g) white vertical lines mark Bruch's membrane. Scale bars, $10 \mu \mathrm{m}$. $(\mathrm{n}=3) \mathbf{h}$, TER of control and CC-HS treated 3D-oBRB $(n=3) . \mathbf{i}, \mathbf{j}$, Confocal images of RPE from 397 3D-oBRB, immunostained with ZO-1 (green) and Hoechst (blue). Arrowhead marks lost ZO-1 signal. Scale 398 bars, $30 \mu \mathrm{m}$. k,l, Depth code of 3D reconstructed confocal images. Color gradient along z-axis $(100 \mu \mathrm{m}$ 399 depth) starting from subRPE zone (red) to the end of choroid (blue); in vehicle-treated 3D-oBRB (k) and 400 in ML228 treated 3D-oBRB (1). Arrowheads mark capillaries in the sub-RPE region. Scale bars, $100 \mu \mathrm{m}$. 401 $\mathbf{m}, \mathbf{n}$, Side view images of CNV, immunostained with ZO-1 (green) and CD31(red). Scale in x-axis, $50 \mu \mathrm{m}$ 402 and in z-axis, $10 \mu \mathrm{m}$. i-n, $(\mathrm{n}=5)$ o, TER measurement on vehicle or ML228 treated 3D-oBRB $(\mathrm{n}=5)$. $\mathbf{p}, \mathbf{q}$ 403 apical (p) and basal (q) VEGF secretion in vehicle and ML228 treated 3D-oBRB (n=4). r, Fluorescence 404 intensity of VEGF staining in cryosectioned 3D-oBRB tissue slice. Quantification of subRPE region (0.0 405 fractional distance) to the bottom of the choroid layer (1.0 fractional distance) $(n=3)$. Statistical significance 406 was attributed to values of $\mathrm{p}<0.05$ as determined by paired t-test $(\mathrm{o}, \mathrm{p}, \mathrm{q})$ or two-way ANOVA and Sidak's 407 multiple comparison test $(\mathrm{g}, \mathrm{h}) .{ }^{*} \mathrm{p}<0.05, * * * \mathrm{p}<0.001, * * * * \mathrm{p}<0.0001$. All error bars indicate STE $408 \quad(\mathbf{g}, \mathbf{0}, \mathbf{p}, \mathbf{q}, \mathbf{r})$. 


\section{Bevacizumab halts CNV in the 3D-oBRB disease model}

416 To validate our CNV model for drug discovery, we asked whether we could replicate the efficacy of an 417 anti-VEGF monoclonal antibody (bevacizumab), used for the treatment of wet $\mathrm{AMD}^{6}$. We co-treated 2D 418 iRPE and 3D-oBRBs with ML228 and a clinical dose $(0.3 \mathrm{mg} / \mathrm{mL})$ of bevacizumab. Bevacizumab had little, 419 if any, effect reversing ML228-induced 2D iRPE atrophy (Figs. 6a-d). In contrast, in the 3D-oBRB 420 bevacizumab co-treatment with ML228 partially recovered RPE epithelial phenotype as confirmed by 421 regained epithelial morphology in ZO-1 immunostained images (arrowheads, Figs. 6e-g) and doubled TER

422 values of ML228+ bevacizumab co-treated tissues (Fig. 6d). 3D reconstruction of tissue cross-sections 423 revealed a noticeable reduction in CNV with bevacizumab and ML228 co-treatment as compared to ML228 424 treatment alone (arrowhead, Figs. 6h-j, movie S6). Image-based quantification of vascular density in each 425 focal plane showed a 3-fold higher $(\mathrm{p}<0.05)$ capillary density within $5 \mu \mathrm{m}$ of the iRPE monolayer in ML228 426 treated samples that were suppressed entirely in bevacizumab and ML228 co-treated samples (Fig. 6k). 427 There was no significant difference in vascular density deeper than 10 microns in the tissue (Figs. 6k, S13a428 c). Overall, these results suggest that hypoxia induced CNV seen in our 3D-oBRB is primarily VEGF 429 induced and can be suppressed by the clinically used drug bevacizumab. All together, these results validate 430 the utility of our tissue for discovering new drugs to treat AMD. 


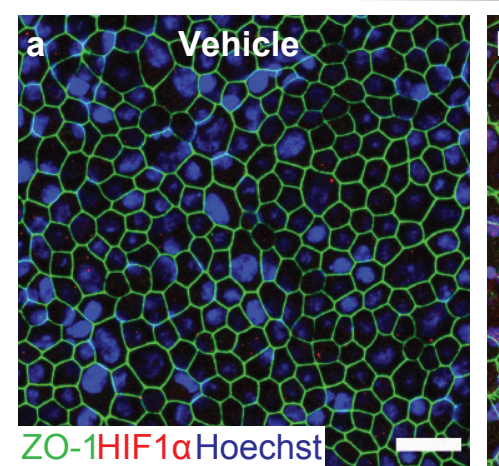

20-1HIF1aHoechst

ш
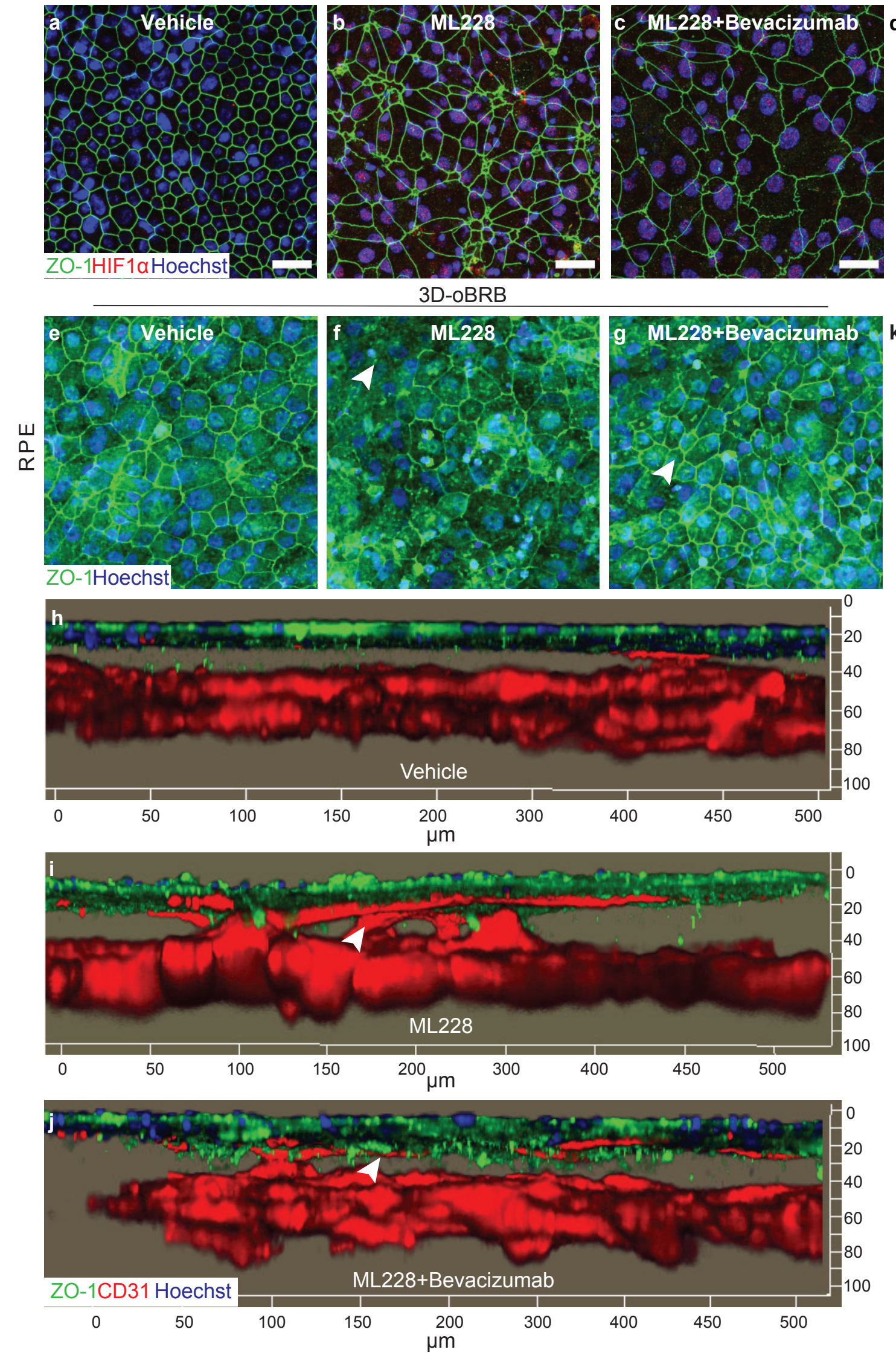

0

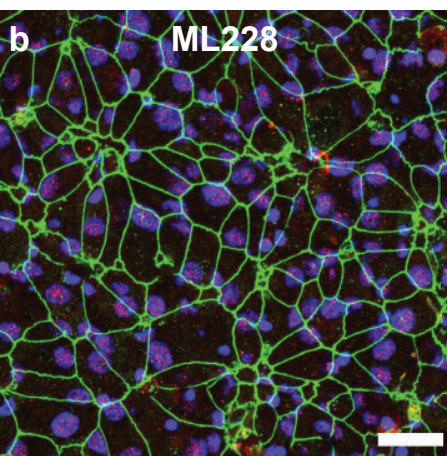

\section{D-oBRB}
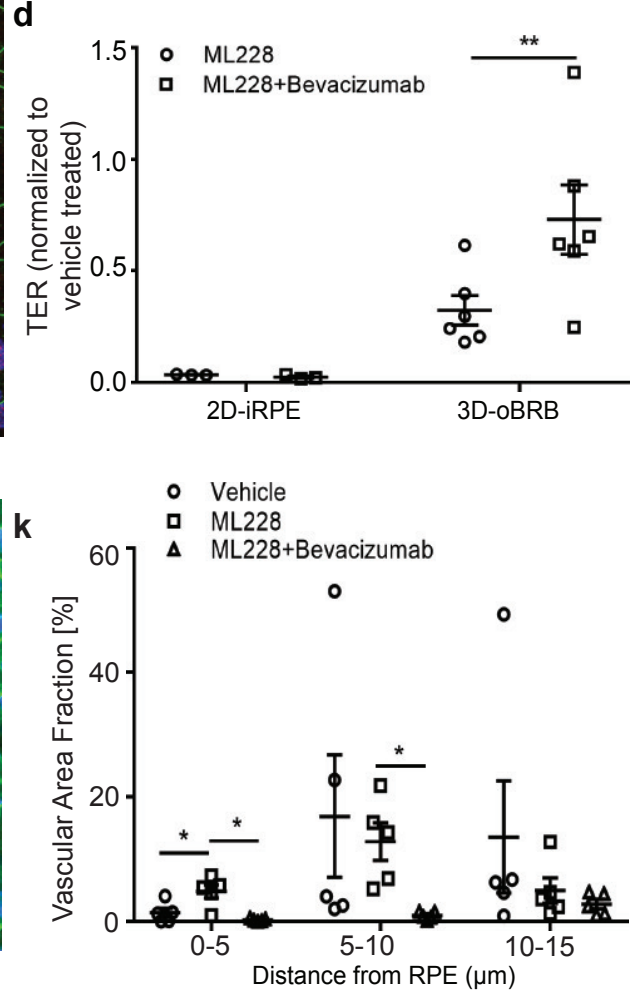
Fig. 6 | Bevacizumab treatment suppresses wet-AMD in 3D-oBRB. a-c, RPE monoculture at 2 weeks

2

56

57

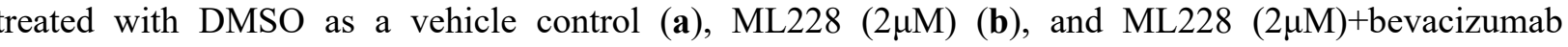
$(0.284 \mathrm{mg} / \mathrm{ml})(\mathbf{c})$, immunostained for HIF-1 $\alpha$ (red), ZO-1 (green), and nuclei stained with Hoechst (blue). Scale bars, $30 \mu \mathrm{m}$. $(\mathrm{n}=3)$. d, TER measurement comparison between 2D-iRPE and 3D-oBRB for ML228 amd ML228+bevacizumab treated samples. TER values were normalized to vehicle treated 3D-oBRBs. (2D-iRPE, $n=3$; 3D-oBRB, n=6). e-g, Maximum intensity projection images of RPE of 3D-oBRB, immunostained with ZO-1 (green) and stained with Hoechst for nuclei (blue). Degenerated and recovered RPE are marked with arrowheads in (f and g). Scale bars, $25 \mu \mathrm{m}$. $\mathbf{h}-\mathbf{j}$, Side view of 3D reconstructed images of vehicle (h), ML228 (i), and ML228+bevacizumab (j) treated 3D-oBRB tissues, immunostained with ZO-1 (green) and CD31(red). Arrowheads in (i) mark hyperproliferating capillaries and in (j) mark retracted capillaries. Scale in $\mathrm{x}$-axis, $50 \mu \mathrm{m}$ and in z-axis, $10 \mu \mathrm{m} . \mathbf{e}-\mathbf{j},(\mathrm{n}=4) . \mathbf{k}$, Vascular area fraction was calculated from CD31 positive area in each z-stack. $(n=5)$. Statistical significance was attributed to values of $\mathrm{p}<0.05$ as determined by unpaired t-test (m) or two-way ANOVA and Sidak's multiple comparison test (d,h,l). $* \mathrm{p}<0.05, * * \mathrm{p}<0.01, * * * * \mathrm{p}<0.0001$. All error bars indicate STE. 3

4

5

(1)




\section{Discussion}

462 3D-oBRB recapitulated key native features that have not been previously replicated in vitro: (1) a fully

463 mature and polarized RPE monolayer with basal infoldings that are critical for metabolite transport and

464 membrane trafficking. Consistently, an increased expression of exosome transport genes was seen in iRPE

465 in the 3D-oBRB. Basal infoldings are lost in diseases like choroideremia and AMD, underscoring the 466 importance of our 3D-oBRB for studying both monogenic and polygenic eye diseases ${ }^{35}$; (2) a Bruch's

467 membrane that mediates RPE and capillary interactions. Its hydraulic conductivity ensures the free flow of 468 nutrients, metabolites and cytokines between the two tissues ${ }^{28}$. With age and in AMD, loss of Bruch's 469 membrane hydraulic conductivity disrupts communication between the two tissues and is thought to be the 470 cause of outer retina degeneration ${ }^{36}$. Our 3D-oBRB replicates degenerative changes in the Bruch's 471 membrane (APOE deposits and angiogenic invasion) and allows the possibility of discovering how such 472 changes contribute to disease; and (3) a capillary-bed with a functional lumen, fenestration, choroid-specific 473 gene expression and ability to integrate upon transplantation. Loss of fenestration is associated with AMD 474 risk-alleles and advanced $\mathrm{AMD}^{18}$. Our model provides direct evidence that fenestration is dependent on the 475 presence of healthy RPE and a possibility of understanding the role of AMD risk-alleles via the use of 476 patient iPSCs.

478 These features were possible due to the introduction of several innovative bioengineering attributes: (1) a 479 biodegradable scaffold provided two bioactive surfaces allowing RPE monolayer growth on one side and 480 bioink printing on the opposite side. Scaffold micropores allowed RPE and ECs to deposit ECM leading to 481 the formation of a Bruch's membrane to recreate a native-like tissue architecture ${ }^{2}$; $(2)$ the mixture of gelatin 482 and fibrin provided a temperature-sensitive hydrogel with high fluidity at room temperature for developing 483 a homogenously concentrated bioink of ECs, pericytes, and fibroblasts and low fluidity at $10^{\circ} \mathrm{C}$ for 484 relatively easier bioprinting. Fibrin maintained the 3D tissue structure until fibroblasts secreted sufficient 485 ECM to provide substrate for a stable capillary-bed; (3) a combination of relevant cell types at appropriate 
ratio: 2:1 fibroblasts to ECs and 1:10 pericytes to ECs allowed easier EC migration and angiogenesis

487 through a native-like ECM structure and sustained long-term capillaries of the correct lumen size $(5-20 \mu \mathrm{m})$.

488 RPE cells provided VEGF for capillary growth, confluency, fenestration, and choroidal phenotype.

490 Our 3D-oBRB constructs recapitulated AMD phenotypes and shed light on disease mechanisms that could 491 not be addressed previously due to the lack of appropriate in vitro model systems. In 3D-oBRB, drusen 492 accumulated within the Bruch's membrane, similar to the observation in AMD eyes ${ }^{2,3}$. This allowed 493 monitoring of drusen impact on Bruch's membrane. RPE atrophy led to choroid degeneration, providing a 494 direct proof of observations made in AMD cadaver eyes that suggested RPE atrophy precedes choroid 495 degeneration. The data from our model suggests that complement inhibition might be a potential therapeutic 496 strategy at an earlier disease stage before the RPE atrophy begins. Our model also provides direct proof 497 supporting previous work that suggested CNV is induced by abnormal VEGF secretion by RPE cells ${ }^{37,38}$.

498 We provide additional insight into CNV by showing higher VEGF secretion induced by RPE hypoxia; and 499 binding of basally secreted VEGF to ECs - combined these data suggest a combination of HIF-1 $\alpha$ inhibitor 500 with a VEGF-receptor blocker will be a more effective in CNV, especially where anti-VEGF agents have 501 failed.

503 Here we have developed a relatively complete model of the oBRB and validated its clinical relevance by 504 demonstrating the efficacy of a clinically used anti-VEGF antibody. A fully-syngeneic 3D-oBRB derived 505 from AMD-patient iPSCs will help provide a more comprehensive understanding of disease pathology and 506 help determine the role of genetics in disease manifestation. 


\section{Donor tissues}

510 Human cadaver eyes were obtained from the Advancing Sight Network (Alabama Eye Blank, Birmingham,

511 AL). Use of cadaver eyes was exempt from the NIH Institutional Review Board approval.

\section{Tissue culture media}

513 RPE Maintenance Media (RPE-MM) components includes basal MEM alpha-modified media (Sigma-

514 Aldrich, Cat\# M-4526, St. Louis, MO) supplemented with N-2 supplement (1\% v/v, Gibco, Cat\# 17502048,

515 Waltham, MA), Glutamine, Penicillin and Streptomycin (1\% v/v, Sigma-Aldrich, Cat\# G1146), Non-

516 essential Amino Acids (5mL, Sigma-Aldrich, Cat\# M7145), a Taurine, Hydrocortisone and Triiodo-

517 Thyronin cocktail dissolved in DPBS (125 mg/10 ug/0.0065 ug, Sigma-Aldrich, Cat\# T-0625/H-0396/T-

518 5516) and heat-inactivated fetal bovine serum (5\% v/v, FBS, Sigma-Aldrich, Cat\# F4135-6X500ML/6505).

519 iPSC Endothelial Cell Media (iCell Media) consists of VascuLife Basal Medium (Thermo Fisher Scientific,

520 Cat\# LL-0003, Waltham, MA), growth factors from the VEGF LifeFactors ${ }^{\circledR}$ Supplement Kit (LifeLine

521 Cell Technologies, Cat\# LS-1020, Frederick, MD), and iCell Endothelial Cells Medium Supplement

522 (Cellular Dynamics International, Cat\# M1019, Madison, WI). Note that fetal bovine serum and gentamycin

523 were excluded from the LifeFactors ${ }^{\circledR}$ supplement kit. All Media was filtered through sterile $0.22 \mu \mathrm{m}$ pore

524 filters before use.

\section{Cell/Tissue Culture}

526 Human placental microvascular pericytes (Angio-Proteomie, Cat\# cAP-0029, Boston, MA) were cultured 527 on $25 \mathrm{~cm}^{2}$ Easy Flasks (Thermo Fisher Scientific, Cat\# 156367) coated with quick coat solution (3mL, 528 Angio-Proteomie, Cat\# cAP-01). Pericytes were cultured in RPE-MM, which is changed every other day. 529 Pericytes were passaged up to three times prior to 3D bioprinting. iCell endothelial cells (Cellular Dynamics International, Cat\# R1022) were cultured on $75 \mathrm{~cm}^{2}$ Easy Flasks (Thermo Fisher Scientific, Cat\# 156499) coated with Fibronectin (Gibco, Cat\# 33016-015). The cells were cultured in the iCell Media with media changes occurring every other day. Cells were passaged at $>70 \%$ confluence by incubating cells in 
534 in iCell complete endothelial medium and centrifuged at $200 \mathrm{RCF}$ for 5 minutes. The cells were passaged 535 up to two times prior to 3D Bioprinting. The adult choroidal fibroblasts were isolated from cadaver human 536 eyes which were procured from Alabama Eye Bank. Fibroblasts were cultured on Primaria tissue culture 537 flasks (Corning, Cat\# 353824, Corning, NY) in RPE-MM.

iPSC derived Retinal Pigment Epithelium (iPSC-RPE) were purchased from Cellular Dynamics International. iPSC-RPE cells were seeded with initial density of $2.5 \times 10^{5}$ cells/well on a vitronectin coated transwell membrane (0.5 mg/mL, Thermo Fisher, Cat\# A31804; Corning, Cat\# 3407). The medium was changed every other day. After two weeks of post-seeding, RPE maintenance media supplemented with

542 Prostaglandin E2 (Tocris Biosciences, Cat\# 363-24-6, Bristol, United Kingdom) at a $100 \mu \mathrm{M}$ concentration

543 were fed to the iPSC-RPE cells for maturation.

\section{Biodegradable scaffold and bioink}

545 Scaffolds used to support 3D-oBRB were constructed by modifying commercially available trans-well inserts (Corning, Cat\# 3407). The permeable membranes included with the inserts were removed and 547 replaced with 12-mm diameter circular sheets of biodegradable PDLGA scaffold (Polysciences, Cat\# 052218-6_1_20_20_55_24, Warrington, PA; Biosurfaces, Boston, MA). Following the removal of the transwell membranes, the transwell frames were dipped into Kwik-Cast silicone gel (World Precision Instruments, Cat\# KWIK-CAST, Sarasota, FL), and the PDLGA scaffolds were attached to the frames. The 551 gel solidified for 5 minutes with the scaffold facing up. Afterward, O-rings (Inner diameter: 10.4mm, Outer 552 diameter: $14.5 \mathrm{~mm}$ ), which were 3-D printed using an Ultimaker2+ 3D Printer (Ultimaker, Utrecht, 553 Netherlands), were treated on its inner surface using additional Kwik-Cast gel and attached to the basal side 554 of the modified transwells. The gel solidified for 10 minutes, permanently attaching the scaffolds to the 555 transwell frame. The scaffolds were coated on the apical side with vitronectin and incubated for an hour at 556 room temperature, followed by an additional hour of incubation at $37^{\circ} \mathrm{C}$. Afterward, vitronectin from the 557 apical side was aspirated and $4 \mathrm{~mL}$ of cell culture grade water was added to the bottom of the scaffold in 
well plates. The scaffolds were then incubated at $37^{\circ} \mathrm{C}$ for 2 hours. Following incubation, the water was aspirated, and the scaffolds were dried overnight.

560

561

562

563

564

565

566

567

568

569

570

571

572

573

574

575

576

577

578

579

580

On the day of bioprinting, a $2.5 \mathrm{mg} / \mathrm{mL}$ fibrinogen solution (Sigma-Aldrich, Cat\# F3879, St. Louis, MO) was prepared in DPBS (Calcium and Magnesium free, Thermo Fisher Scientific, Cat\# 14190144, Waltham, MA) to encapsulate cells during "choroid" printing. Aprotinin (0.075 U/mL, Sigma, Cat\# A4529) was added to the fibrinogen solution, and the entire solution was filtered through a sterile $0.22 \mu \mathrm{m}$ filter. Following filtration, a $300 \mathrm{mg}$ sample of Novogel 2.0 (Organovo 3D Bioprinting Solutions, Cat\# NVG-2.0, San Diego, CA) was carefully added to $5 \mathrm{~mL}$ of the fibrinogen solution. The solution was incubated in a $37^{\circ} \mathrm{C}$ water bath to dissolve the Novogel powder. After dissolution, the completed $2.5 \mathrm{mg} / \mathrm{mL}$ fibrinogen solution was incubated at $37^{\circ} \mathrm{C}$ for 1 to 2 hours prior to cell encapsulation. A $5.0 \mathrm{mg} / \mathrm{mL}$ fibrinogen solution was prepared identically to the $2.5 \mathrm{mg} / \mathrm{mL}$ solution above without the addition of Novogel powder. This solution was placed at $4^{\circ} \mathrm{C}$ until bioprinting.

\section{Bioprinting of "choroid" and 3D-oBRB maturation}

Following assembly of the scaffolds and immediately before bioprinting, the scaffolds were treated in a Plasma Etch PE-50 oxygen-plasma etcher at maximum power (150W, Plasma Etch, Inc., Carson City, NV) for 30 minutes under $10 \mathrm{CC} / \mathrm{min}^{2}$ flow to sterilize and improve surface hydrophilicity.

Fibroblasts, endothelial cells, and pericytes were trypsinized from cell culture flasks as described previously. Cells were encapsulated at the following densities in the $2.5 \mathrm{mg} / \mathrm{mL}$ fibrinogen solution: 1.0 to $1.2 \times 10^{7}$ Fibroblasts $/ \mathrm{mL} ; 5.0$ to $7.0 \times 10^{6}$ Endothelial cells $/ \mathrm{mL}$; and 5.0 to $7.0 \times 10^{5}$ Pericytes $/ \mathrm{mL}$. The three cell types were combined and centrifuged at $500 \mathrm{RCF}$ for 4 minutes before resuspension in the $2.5 \mathrm{mg} / \mathrm{mL}$ fibrinogen solution. Resuspension and mixing were conducted using a sterile spatula (Corning, Cat\# 3004) to prevent air bubble formation in the suspension. The cell suspension transferred to a sterile Hamilton Gastight syringe without air bubbles (Hamilton, Cat\# 1750). The syringe was then sealed and placed at $4^{\circ} \mathrm{C}$ 

Diego, CA) was used to extrude the solution onto the biodegradable polymer scaffolds.

583 After the structure was printed, $360 \mu \mathrm{L}$ of $5 \mathrm{mg} / \mathrm{mL}$ fibrinogen solution was added to a $40 \mathrm{uL}$ aliquot of 10 $584 \mathrm{U} / \mathrm{mL}$ thrombin. $170 \mathrm{uL}$ of the $5 \mathrm{mg} / \mathrm{mL}$ fibrin gel with thrombin was then added directly to the printed structure to form a supporting fibrin gel structure between printing lanes. It solidified for 15-20 minutes. Printing Medium, which consists of a 1:2 ratio of iCell media and serum-free RPE-MM that was supplemented with $1.67 \%$ heat-inactivated FBS, thrombin $(0.5 \mathrm{U} / \mathrm{mL}$, Sigma, Cat\# T6884), rh ANG-1 (100ng/mL, R\&D Systems, Cat\# 923-AN, Minneapolis, MN), VEGF (85 ng/mL, R\&D systems, Cat\# 293VE-500), and aprotinin, was then added to the well (0.5 mL apically, and $3.5 \mathrm{~mL}$ basally) and placed at room temperature 2 hours. The samples were then placed in a $37^{\circ} \mathrm{C}, 5 \% \mathrm{CO}_{2}$ incubator overnight.

Bioprinted 3D-oBRB tissues consist of multiple cell types such as endothelial cells, pericytes, fibroblasts, and iPSC-RPE and were incubated under a complex medium changing schedule (Table 1). RPE cells were seeded on the apical side of the PDLGA scaffold at day 7 after bioprinting. $2.5 \times 10^{5}$ RPE cells were added to each bioprinted tissue in suspension of RPE-MM. The RPE growth and maintenance media were used according to table S1. Printing Media was applied to the apical and basal sides of the tissue on the day of the print. Tissues were then fed on the apical and basal sides using Vascular Development Media 597 (VDM), which has the same composition as Printing Media but with thrombin removed, from day 2 to day 7. On day 7, the apical sides of the tissues were fed using 5\% RPE, while the basal sides of the tissues were fed using Vascular Growth Media with Aprotinin (VGM+AP), which excludes ANG-1 and VEGF from VDM. On day 14, the apical sides of the tissues continued to be fed using 5\% RPE, while basal sides of the tissues were fed using VGM which excludes Aprotinin from VGM+AP. On day 21, media conditions were identical to the previous week, except that the 5\% RPE medium was supplemented with Prostaglandin E2 at a $100 \mu \mathrm{M}$ concentration on the apical side. From Day 29 onward, media conditions were identical to the previous week, except that Vascular maintenance media (VMM), which excludes VEGF completely, replaced VGM medium on the basal side. 


\section{Rat Transplantation}

607 The tissue was gently wash with BSS+ (Alcon Inc, Cat\# 00065079515) and a thin layer of Healon PRO

608 (Johnson \& Johnson Vision Care Inc) was used to protect it during manipulation. A 1.5mm (Acuderm Inc 609 Cat\# P1525) puncher was used to cut the dose to deliver from the cultured tissue. RNU rats from Charles 610 River Laboratories, Inc., Wilmington, MA were used in this study. The animals $(n=4$, males, between 250 611 and $300 \mathrm{~g})$ were anesthetized with IP injection of Ketamine (65 mg/kg, VetONE Inc, Cat\#13985-702-10) 612 and $7.5 \mathrm{mg} / \mathrm{kg}$ Xylazine (7.5 mg/kg, Akorn Inc, 59399-111-50). Animals were then placed on a shaped 613 foam holder and the body was covered to avoid body temperature drop. Isoflurane was administered in 614 mask, $1 \%$ with an oxygen flow rate of $300 \mathrm{ml} / \mathrm{min}$, to maintain anesthesia. Eyes were exposed and secured 615 by a suture tide around the eyeball (Figure X). Proparacaine (0.5\%, Novartis, Cat\# 61314-016-01) was used 616 as local anesthetic and the pupil were dilated with Phenylephrine (Paragon Biotek, 4270210215) and 617 Tropicamide (Novartis, Cat\# T2018-39) eye drops. GenTeal lubricant eye gel (Alcon, Cat\# US-GNT-VLC618 2000008) and a glass cover slip were used as a lens substitute to visually access the back of the eye. A $6192.2 \mathrm{~mm}$ knife was used for sclerotomy, vitreous was replaced with 2\% Hyaluronic acid (HA) (Sigma Aldrich, 620 Cat\# H7630) and the retina was detached with 0.5\% HA (Figure X). The tissue was then delivered in the 621 subretinal space with end-grasping ophthalmic forceps (Figure X). The retina was flattened with $2 \%$ HA. 622 The suture used to expose the eye was removed and topic Neo-Poly-Bac (Bausch \& Lomb, Cat\# 623 IWM044525) ophthalmic ointment was used to prevent infections. Animals were kept in a warm, dark and 624 quiet environment until complete recover from anesthesia, then were single caged until the end of the 625 experiment. Two weeks after surgery animals were euthanized by $\mathrm{CO}_{2}$ overdose and perfused with 1,1'626 dioctadecyl-3,3,3',3'-tetramethylindocarbocyanine perchlorate (DiI) (Sigma Aldrich, Cat\# 42364) a 627 lipophilic carbocyanine dye, which incorporates into endothelial cell membranes upon contact (Li et al., 628 2008). The dye was prepared and administered as previously described by Li, followed by $4 \%$ PFA 629 perfusion. Eyes were collected and fixed in 4\% PFA overnight. 
631 We modeled wet and dry AMD on four-week-old tissue using a chemical stressor or complement competent 632 human serum, respectively. For Wet-AMD, ML228 $(2 \mu \mathrm{M})$ was introduced to the apical side of RPE and 633 treated for four days, and Bevacizumab $(0.284 \mathrm{mg} / \mathrm{mL}$, NIH clinical center) was treated with ML228 after

634 the four days of the ML228 treatment. ML228 was treated every day and Bevacizumab was treated every 635 other day. For Dry AMD, complement competent human serum (5\%) was treated to both apical and basal 636 side of the tissue with everyday media change. The total inducing period for both AMD models is two 637 weeks.

Trans-Epithelial Resistance measurements and fixation

639 The junctional integrity of 3D-oBRB tissues or iPSC-RPE monolayers were examined prior to fixation. 640 Trans-Epithelial Resistance (TER) was measured using an EVOM2 Epithelial Volt/ohm meter (World 641 Precision Instruments, Cat\# 300523) and an Endohm-245NAP well container (World Precision 642 Instruments). Afterward, tissue samples were incubated in $4 \%$ Paraformaldehyde in $1 \mathrm{x}$ PBS at $4^{\circ} \mathrm{C}$ 643 overnight for fixation and were washed three times for 10 minutes in 1x PBS. At this point, whole tissue 644 samples were either Cryosectioned (See: Cryosectioning) or Paraffin-sectioned (See: Paraffin-sectioning) 645 or immunostained (See: Immunostaining). If whole tissue samples were to be immunostained directly, 646 samples were permeabilized using in 0.5\% Triton X-100 (Thermo Fisher Scientific, Cat\# 85112) solution 647 in 1x PBS. Samples were punched out using a biopsy punch,transferred to the 24 well and blocked in 5\% 648 goat serum, $0.1 \%$ Triton X-100 and 1\%BSA in $1 \mathrm{x}$ PBS for 1 hour at room temperature. Afterward, the 649 samples were washed three times for 10 minutes in 1x PBS.

\section{Microscopy}

653 Time lapse images of GFP labeled endothelial cells were taken by aEVOS Auto FL (Thermo Fisher 654 Scientific, NY). Time-course immunofluorescent images for quantification were taken by using Leica TCS- 
SP8 (Leica, Wetzlar, Germany) and Zeiss LSM710 (Zeiss, Oberkochen, Germany) confocal microscopes.

$6563 \mathrm{D}$ reconstructed images with high magnification (x25) were taken by SP8 confocal microscope (Leica).

657 Transmission electron microscopy with glutaraldehyde fixed samples was taken by the NEI core facility 658 using a JEM-1010 electron microscope (JEOL, Peabody, MA).

\section{Histology and immunofluoresence}

661 Cryosectioning: Fixed tissue samples were punched from transwell inserts using a 10-mm biopsy punch 662 and incubated in 15\% sucrose, followed by $30 \%$ sucrose until the water was sufficiently removed from the 663 tissue sample (approximately 1-hour incubation per solution). Rat Ocular Tissue was extracted from 664 animals, and then flash frozen. Afterward, the tissues were embedded in Optical Cutting Temperature 665 Embedding Medium (Thermo Fisher Scientific, Cat\# 23-730-571) and frozen using dry ice for a minimum 666 of 90 minutes. The samples were stored in $-80^{\circ} \mathrm{C}$ until sectioning. The tissues were cut to to $12-\mu \mathrm{m}-$ 667 thickness slices and loaded onto glass slides using a cryostat (Leica, Germany) at $-30^{\circ} \mathrm{C}$ cutting temperature 668 and $-15^{\circ} \mathrm{C}$ sample head temperature. Prior to staining, tissue slices were heated on a hot plate to $37^{\circ} \mathrm{C}$ for 66930 minutes to secure the section to the glass slide. The tissues were then either H\&E stained using an 670 automated H\&E Staining machine (Bond RX; Leica, Germany), or immunostained. During 671 immunostaining, the slices were blocked and permeabilized using 0.5\% Triton X-100 (Thermo Fisher 672 Scientific, Cat\# 85112) prior to the application of primary and secondary antibodies.

673 Paraffin Sectioning: Fixed samples were permeabilized for 30 minutes in $0.5 \%$ Triton X-100 (Thermo 674 Fisher Scientific, Cat\# 85112). After permeabilization, samples were punched out from transwell inserts 675 using a 10-mm biopsy punch and stored in 24-well plates. Samples were then blocked in 5\% goat serum, $6760.1 \%$ Triton X-100 and 1\%BSA in 1x PBS for 1 hour at room temperature. Afterward, the samples were 677 washed three times for 10 minutes in $1 \mathrm{x}$ PBS. 
Vibratome Sectioning: Fixed 3D-oBRB and Rat Ocular tissues were embedded in 6.8\% w/v Type 7-A Agarose (Sigma-Aldrich, Cat\# A0701) and cut into $100 \mu \mathrm{m}$ sections on the Leica VT 1200S vibratome (Leica, Wetzlar, Germany). Sectioned samples were blocked, permeabilized, and washed prior to staining as described previously

Immunostaining: Primary antibodies were diluted in $1 \mathrm{X}$ PBS containing $0.1 \%$ Triton-X-100 and 1\% BSA and 3D-oBRB tissues, or iPSC-RPE monolayers were incubated at $4{ }^{\circ} \mathrm{C}$ overnight. Primary antibodies included Mouse anti-PLVAP (FELS, 1:50, Abcam, Cat\# AB81719); Rabbit anti-CD31 (1:50, Abcam, Cat\# AB28364); Mouse anti CD31 (1:50, Agilent, Cat\# M0823); Rabbit anti-Laminin (1:50; Abcam, Cat\# AB11575); Mouse anti E-cadherin (1:100; Abcam; Cat\# AB40772); mouse anti-ZO-1 (1:100; Thermo Fisher Scientific, Cat\# 33-9100); rabbit anti-ZO-1 (1:50; Thermo Fisher Scientific, Cat\# 61-7300) Mouse anti-Collagen-IV (1:50; Abcam, Cat\# AB6311); Rabbit anti-Elastin (1:50 Abcam, Cat\# AB21610); Rabbit anti-APOE (1:50; Abcam, Cat\# AB52607), Rabbit anti-VEGF (1:50; Thermo Fisher Scientific, Cat\# P807), Mouse anti-STEM121 (1:100; Takara Bio, Cat\# Y40410), Rabbit anti-VWF (1:100; Dako, Cat\#GA527612) and Alexa-Fluor ${ }^{\mathrm{TM}}$ 647-Phalloidin (26 nM, Thermo Fisher Scientific, Cat\# A22287). Blocking controls were performed using primary antibody buffers without the use of primary antibodies. Samples were then washed three times for 10 minutes in 1x PBS. Secondary antibodies were diluted (PBS containing $0.1 \%$ Triton-X-100 and 1\% BSA). Secondary antibodies included Alexa-fluor ${ }^{\mathrm{TM}}$ Goat anti Rabbit 647 (Invitrogen, Cat\# A21244); Goat anti Rabbit 594 (Life Technologies, Cat\# A11012); Goat anti Rabbit 555 (Invitrogen, Cat\# A32732); Goat anti-Rabit 488 (Invitrogen, Cat\# A11008); Goat anti-mouse 647 (Invitrogen, Cat\# A32728); Goat anti-mouse 595 (Invitrogen, Cat\# A11032); Goat anti-mouse 555 (Invitrogen, Cat\# A21422); Goat anti Mouse 488 (Invitrogen, Cat\# A32723). Secondary antibodies were incubated with Hoescht 33342 nuclear stain (1:2000) in the dark at room temperature for 4 hours. Samples were washed 3 times for 10 minutes in 1xPBS. Cryosectioned, Parafilm-sectioned and whole tissue samples were mounted using Fluoromount G (Southern Biotech, Cat\# 0100-01, Birmingham, AL) following immunostaining. 


\section{Quantification}

704 Angiogenesis quantification: Fluorescent images of vasculature at 4, 6, and 8 days after bioprinting, either 705 non-treated or treated with varying doses of VEGF were quantified using MATLAB version 2019b 706 (Mathworks, Natick, MA). Six line probes, numbered 1 to 6, were created in the acellular spaces between 707 printed stripes. Fluorescence intensity peaks with a threshold (100 in 8-bit gray scale) that overlapped with 708 the line probes were quantified in each image (Fig. S2). The counted peaks from each line number were averaged per each vascular micrograph, representing the "edges" to "centers" of the acellular space. To determine the directionality and persistence of blood vessel migration into the acellular regions, an angiogenesis index was created to quantify mean blood vessel length perpendicular to the printed stripes.

712 Confocal images were processed as maximum intensity projection images and the angiogenesis index was measured using MATLAB (Mathworks). Briefly, regions of interest were defined in acellular spaces as a space within 250 pixels of a line probe generated through the center of the spaces. Acellular spaces that overlapped with out-of-focus endothelial cell clusters were excluded from analysis (Fig. S5b). Blood vessels were defined as objects that were greater than 20 pixels in length and expanded to within a mean distance of 85 pixels of the line probe. Angiogenesis index was calculated using maximum ferret angle of each blood vessel relative to the line probe and maximum ferret diameter of each blood vessel [equation 719 (1)].

722 Immunostaining images of CD31 and FELS were analyzed using ImageJ to quantify the vascular networks 723 in 3D-oBRB tissues. Any CD31-labeled images taken one week after bioprinting were contrasted with 724 isolating the brightest $80 \%$ of pixels (Leica TCS-SP8 and Zeiss LSM710) from the background. For the 725 rest of the time points, CD31-labelled images were contrasted with isolating the brightest $20 \%$ of pixels 726 from the background. FELS-labeled images were contrasted with separating the brightest 5\% of pixels from 727 the background at all time points. The area ratios were taken between FELS-positive area/image and the 

FELS.

VEGF Immunostaining Quantification: Z-stacks of the VEGF-A-stained cryosectioned tissue slices were analyzed using ImageJ. The images were converted into maximum intensity projection images before quantification. For image quantification, a Gaussian Blur Filter (radius 4.0 px) was applied to reduce noise in the images. The average pixel brightness along the thickness of the slice was quantified for each slice to identify areas of high VEGF concentration in the slices. Phalloidin 647 staining was used to identify the apical (RPE) and basal (choroid) ends of the tissue slices.

Cytokine Quantification: To quantify the cytokine secretion from iPSC-RPE monolayers and 3D-oBRB tissues, apical and basal media were collected at 48-hour, 96-hour, and 2-week timepoints and frozen at $80^{\circ} \mathrm{C}$ until the assay was performed. Once thawed, the samples were centrifuged at $1400 \mathrm{RPM}$ for 5 minutes to remove possible cell debris from sample supernatant. The assay was carried out using a Millioplex MAP Kits per the manufacturer's instructions (Millipore-Sigma, Cat\# HCYTOMAG-60K) analyzed at the NIH Flow Cytometry Core Facility.

Vascular Density Quantification: 3D reconstruction of tissues was conducted on confocal images taken with 20x or 25x magnification, and area fractions of CD31 expression were calculated in each focal plane. For estimating distance from RPE region, beginning focal plane of vasculature was manually determined by comparing to z-stack images with ZO-1 expression by RPE. Thresholding was performed on z-stack images of the CD31 channel by Otsu thresholding method in ImageJ. Step size of the distance from RPE was determined as $5 \mu \mathrm{m}$ to adjust z-step size differences between samples.

Quantification of Extracellular Matrix Deposition: RPE monoculture and 3D-oBRB tissues in 24 transwell plates were fixed with 4\% PFA after 5 weeks from RPE seeding on transwell membrane or bioprinted "choroid". The z-stacks of confocal images from the apical side of RPE to the basal side of RPE 
751 were taken with the same microscope settings among the treatment groups. Mean intensity of Elastin and 752 Collagen IV expression were measured using Image J.

\section{Single cell RNA sequencing}

Endothelial cell Isolation from 3D-oBRB tissues: The endothelial isolation was performed by digesting the $3 \mathrm{D}$-oBRB tissues. The digestion solution was created using $1.5 \mathrm{mg} / \mathrm{mL}$ Collagenase II (Gibco, Cat\# 17101-015), 0.5 mg/mL DNAse I (Worthington Biochemical Corporation, Cat\# 54D14897, Lakewood, NJ) and $0.2 \mathrm{mg} / \mathrm{mL}$ Dispase I (Sigma, Cat\# D4818) in PBS. Tissues were removed with a sterile scalpel, placed in a $50 \mathrm{~mL}$ conical tube. Digestion solution was added (approximately $10 \mathrm{~mL} / 6$ tissues) and the samples were rocked on an orbital shaker at $37^{\circ} \mathrm{C}$ for 30 minutes. Following the incubation, the suspension is given $20 \mathrm{~mL}$ of media and is then centrifuged at $1000 \mathrm{RPM}$ for 5 minutes. Endothelial cells were then purified using magnetic assisted cell sorting (Miltenyl Biotec, Cat\# 130-042-401) protocol supplied by Miltenyl Biotec, counted, and analyzed with assistance from the NCATS Stem Cell Translation Laboratory.

Single-cell RNA Library Preparation: Single-cell suspensions were loaded on a Chromium Controller (10X Genomics) to generate single-cell gel bead-in-emulsions (GEMs) and barcoding. GEMs were transferred to PCR 8-tube strips, and GEM-reverse transcription was performed in a C1000 Touch Thermal Cycler (BioRad). GEMs were lysed in recovery buffer, and single-stranded cDNA was cleaned up using silane DynaBeads (Thermo Fisher Scientific). cDNA was amplified in a C1000 Touch Thermal Cycler (BioRad). Amplified cDNA was cleaned up using the SPRIselect Reagent (Beckman Coulter). Post cDNA amplification QC and quantification were done using a High Sensitivity D5000 ScreenTape Assay (Agilent) on a 4200 TapeStation System (Agilent). Library Construction was done by fragmentation at $32{ }^{\circ} \mathrm{C}$ for 5 min, end repair, and A-tailing at $65^{\circ} \mathrm{C}$ for $30 \mathrm{~min}$. Post fragmentation, end repair, and A-tailing doublesided size selection were done using the SPRIselect Reagent (Beckman Coulter). Adaptor ligation was done at $20{ }^{\circ} \mathrm{C}$ for 15 min. Post ligation cleaned up using the SPRIselect Reagent (Beckman Coulter). Sample 
775 indexing was done using the i7 Sample Index Plate (Chromium) in a C1000 Touch Thermal Cycler 776 (BioRad). Post sample index PCR double sided size selection done using the SPRIselect Reagent (Beckman 777 Coulter). Post library construction quantification was done using a High Sensitivity D1000 ScreenTape 778 Assay (Agilent) on a 4200 TapeStation System (Agilent). Sequencing libraries were quantified by 779 quantitative PCR using the KAPA library quantification kit for Illumina platforms (KAPA Biosystems) on 780 a QuantStudio 12K Flex Real-Time PCR System (Thermo Fisher Scientific). Libraries were loaded on an 781 Illumina HiSeq 3000 using the following: 98bp Read1, 8bp i7 Index, and 26bp Read2.

782 The cellranger software package from 10X Genomics, Inc. (version 3.0.1) was used to process raw BCL 783 files from single-cell sequencing as follows. This work used the computational resources of the NIH HPC 784 Biowulf cluster (http://hpc.nih.gov). Demultiplexing and FASTQ generation were done with the mkfastq 785 command, and the count command created gene expression matrices. Dense matrices were created with the 786 mat2csv command. Embryonic stem cell and iPSC lines were analyzed in the Seurat R package (Stuart et al. 2018 https://www.biorxiv.org/content/10.1101/460147v1; Seurat 2.3.4; R 3.5.2). Further data visualizations were made in $\mathrm{R}$ and with the ggplot2 package (3.1.0) (Table S2). Gene set enrichment analysis was performed using GSEA v4.0.3 (Broad Institute, Inc., MIT), the gene set databases C2 and C5 (MSigDB v6.2), which are the curated and Gene Ontology sets, and EnrichR ${ }^{20}$. Endothelial and RPE single cell RNA-Seq phenotypes for $2 \mathrm{D}$ vs $3 \mathrm{D}$-oBRB cells were coded as 0 and 1 respectively for a test of enrichment by ranked differential gene expression. Significantly enriched phenotypes were selected based on whether there was a nominal $\mathrm{P}$ value of $<0.5$ and a false discovery rate $<25 \%$ between $2 \mathrm{D}$ and $3 \mathrm{D}$ oBRB datasets. Log2 ratio of classes was used for the ranking metric and otherwise default parameters were used. When comparing cytokine gene expression levels to cytokine detection levels in media, we extracted expression data was extracted for gene symbols that corresponded to the Luminex cytokine detection 797 Assays performed previously. Only apical media was considered in these results. Only RPE gene expression 798 data was considered in these results. 
801 Gene List Construction: The ECM gene list was created by finding the gene ontology annotation for 802 "extracellular matrix" ( http:/www.informatics.jax.org/go/term/GO:0031012), then narrowing that list to 803 those genes that are also RPE characteristic genes ${ }^{27}$ and genes found in the CYCLOPS database ${ }^{22}$ to be 804 expressed in the tissues "RPE fetal" and "RPE adult" at levels $>2 x$ levels that were found in at least 70 805 other tissues in the database. Selected genes must also exceed a minimum TPM threshold of 100. Afterward, 806 the gene list was reduced to those pertaining to ECM proteins, matrix metalloproteinases, tissue-inhibitor 807 matrix metalloproteinases, and ECM crosslinking proteins. Endothelial cell signature genes were derived 808 from literature searches related to choroidal maturation ${ }^{21}$, arterial and venous specification ${ }^{19}$, as well as 809 angiogenesis ${ }^{23}$.

810 PCA Cluster Analysis: Statistical comparisons between 2D and 3D-oBRB gene expression and Gene 811 Enrichment analysis were performed in RStudio using the Seurat Gene Expression Analysis Package) and 812 EnrichR. Single Cell samples were filtered to exclude samples with less than 200 unique RNA features, 813 more than 6,000 unique RNA features, and more than 5\% mitochondrial genome content. Afterward, 814 samples were clustered using PCA (15 principal components analyzed) followed by tSNE. Endothelial cell 815 clusters were identified using the Louvain algorithm at 0.2 resolution and RPE cell clusters were identified 816 using 0.6 resolution. Gene enrichment was determined using the FindAllMarkers Seurat function. Genes in 817 clusters with greater than 1.5-fold upregulation over the cell population average were processed in EnrichR 818 to identify enriched biological processes (GO Biological Process 2018). Biological processes in clusters 819 did not have 25 sufficiently upregulated genes were determined using comparisons with similar culture 820 formats (i.e. $2 \mathrm{D}$ vs $2 \mathrm{D}$ or $3 \mathrm{D}-\mathrm{oBRB}$ vs $3 \mathrm{D}-\mathrm{oBRB}$ ) only.

\section{Statistical Analysis}

822 All the data analyzed were unpaired (that is, the samples were independent from each other). Before 823 conducting multiple comparison tests, the Brown-Forsythe test was performed to determine the 824 homogeneity of variance between the datasets. One-way and Two-way ANOVA was performed prior to 825 any post-hoc statistical comparison. To compare multiple datasets, Tukey's multiple comparisons test was 
used as a single-step multiple comparison procedure to find means significantly different from each other.

827 The Bonferroni post-hoc test was used to detect significant differences in the Line-Probe Angiogenesis

828 quantifications. To compare datasets with a vehicle control, Dunnett's test was used to find means

829 significantly different from the control. Student's T-Test was used to find significant differences between

830 two single conditions. All statistical tests were two-tailed (two-sided tests). All statistical analyses were

831 performed using Graphpad Prism version 8.2.0 for Windows (GraphPad Software, San Diego, CA) or

832 Microsoft ${ }^{\circledR}$ Excel ${ }^{\circledR}$ for Microsoft 365 MSO (16.0.13001.20266) 32-bit (Microsoft, Redmond, WA). P <

8330.05 was considered significant. Variances between each group of data were represented by the standard

834 deviation unless otherwise stated. Each statistical analysis method was indicated in individual quantification

835 sections. Comparisons between ANG-1 and treated and non-treated conditions were performed using

836 individual Sidak's Multiple Comparisons Tests on the day 4, 6, and 8 timepoints. Statistical comparisons

837 between 2D and 3D-oBRB cytokine detection were performed using Sidak's Multiple Comparisons Test

838 for each assayed gene. Calculations were performed using Graphpad Prism ver. 8.2.0 (GraphPad Software,

839 Inc). Sample sizes to ensure adequate power were as follows: initial angiogenesis formation between printed

840 structures, $\mathrm{n}=5$ tissue replicates; ANG-1 modulating angiogenesis, $\mathrm{n}=4$ tissue replicates;

841 Immunofluorescence of ECM proteins, $\mathrm{n}=3$; Outer-BRB maturation by RPE and printed choroid, $\mathrm{n}=3$

842 tissue replicates; Bevacizumab treatment on choroidal neovascularization model, $\mathrm{n}=3$ tissue replicates per

843 condition; APOE deposition and dry AMD, $\mathrm{n}=3$ tissue replicates per condition; Single Cell RNASeq, $\mathrm{n}=$

8443012 2D RPE cells, $\mathrm{n}=4380$ 3D-oBRB RPE cells, $\mathrm{n}=5369$ 2D Endothelial cells, $\mathrm{n}=1294$ 3D-oBRB

845 Endothelial cells. Samples were excluded from analysis if they were determined to be outliers through

846 Grubbs' outlier test.

847 Data availability

848 The authors declare that all data supporting the findings of this study are available within the article and its

849 Supplementary Information files or are available from the corresponding author upon request. 


\section{References}

8521 Wong, W. L. et al. Global prevalence of age-related macular degeneration and disease burden projection for 2020 and 2040: a systematic review and meta-analysis. Lancet Glob Health 2, e106116, doi:10.1016/S2214-109X(13)70145-1 (2014).

8552 McLeod, D. S. et al. Relationship between RPE and choriocapillaris in age-related macular 856 degeneration. Invest Ophthalmol Vis Sci 50, 4982-4991, doi:10.1167/iovs.09-3639 (2009).

8573 Bhutto, I. \& Lutty, G. Understanding age-related macular degeneration (AMD): relationships between the photoreceptor/retinal pigment epithelium/Bruch's membrane/choriocapillaris complex. Mol Aspects Med 33, 295-317, doi:10.1016/j.mam.2012.04.005 (2012).

8604
doi:10.1016/j.brainres.2015.12.011 (2016).

Farecki, M. L. et al. Characteristics of type 1 and 2 CNV in exudative AMD in OCT-Angiography. Graefes Arch Clin Exp Ophthalmol 255, 913-921, doi:10.1007/s00417-017-3588-y (2017).

8656 Kovach, J. L., Schwartz, S. G., Flynn, H. W., Jr. \& Scott, I. U. Anti-VEGF Treatment Strategies for Wet 866 AMD. J Ophthalmol 2012, 786870, doi:10.1155/2012/786870 (2012).

8677 Barben, M., Samardzija, M. \& Grimm, C. The Role of Hypoxia, Hypoxia-Inducible Factor (HIF), and 868 VEGF in Retinal Angiomatous Proliferation. Adv Exp Med Biol 1074, 177-183, doi:10.1007/978-3869 319-75402-4_22 (2018).

8708 Campbell, M. \& Humphries, P. The blood-retina barrier: tight junctions and barrier modulation. $871 \quad$ Adv Exp Med Biol 763, 70-84 (2012).

8729 Nickla, D. L. \& Wallman, J. The multifunctional choroid. Prog Retin Eye Res 29, 144-168, 873 doi:10.1016/j.preteyeres.2009.12.002 (2010). 
87410 Hoshino, A., Chiba, H., Nagai, K., Ishii, G. \& Ochiai, A. Human vascular adventitial fibroblasts 875 contain mesenchymal stem/progenitor cells. Biochem Biophys Res Commun 368, 305-310, 876 doi:10.1016/j.bbrc.2008.01.090 (2008).

87711 Orlova, V. V. et al. Generation, expansion and functional analysis of endothelial cells and pericytes 878 derived from human pluripotent stem cells. Nat Protoc 9, 1514-1531, 879 doi:10.1038/nprot.2014.102 (2014).

88012 Sharma, R. et al. Clinical-grade stem cell-derived retinal pigment epithelium patch rescues retinal 881 degeneration in rodents and pigs. Sci Transl Med 11, doi:10.1126/scitranslmed.aat5580 (2019).

88213 Shiihara, H. et al. Quantitative analyses of diameter and running pattern of choroidal vessels in 883 central serous chorioretinopathy by en face images. Sci Rep 10, 9591, doi:10.1038/s41598-02066858-1 (2020).

88514 Lamalice, L., Le Boeuf, F. \& Huot, J. Endothelial cell migration during angiogenesis. Circ Res 100, 886 782-794, doi:10.1161/01.RES.0000259593.07661.1e (2007).

88715 Maminishkis, A. et al. Confluent monolayers of cultured human fetal retinal pigment epithelium 888 889 exhibit morphology and physiology of native tissue. Invest Ophthalmol Vis Sci 47, 3612-3624, doi:10.1167/iovs.05-1622 (2006).

89016 Campochiaro, P. A., Jerdon, J. A. \& Glaser, B. M. The extracellular matrix of human retinal pigment 891 epithelial cells in vivo and its synthesis in vitro. Invest Ophthalmol Vis Sci 27, 1615-1621 (1986).

89217 Choi, W. et al. Choriocapillaris and choroidal microvasculature imaging with ultrahigh speed OCT 893 angiography. PLoS One 8, e81499, doi:10.1371/journal.pone.0081499 (2013).

89418 Grebe, R. et al. Ultrastructural analysis of submacular choriocapillaris and its transport systems in $895 \quad$ AMD and aged control eyes. Exp Eye Res 181, 252-262, doi:10.1016/j.exer.2019.02.018 (2019).

89619 dela Paz, N. G. \& D'Amore, P. A. Arterial versus venous endothelial cells. Cell Tissue Res 335, 5-16, 897 doi:10.1007/s00441-008-0706-5 (2009). 
898

899

900

901

902

903

904

905

906

907

908

909

910

911

$912 \quad 25$

913

$914 \quad 26$

915

916

$917 \quad 27$

918

919

920

921

20 Kuleshov, M. V. et al. Enrichr: a comprehensive gene set enrichment analysis web server 2016 update. Nucleic Acids Res 44, W90-97, doi:10.1093/nar/gkw377 (2016).

21 Songstad, A. E. et al. Generating iPSC-Derived Choroidal Endothelial Cells to Study Age-Related Macular Degeneration. Invest Ophthalmol Vis Sci 56, 8258-8267, doi:10.1167/iovs.15-17073 (2015).

22 Swamy, V. \& McGaughey, D. Eye in a Disk: eyelntegration Human Pan-Eye and Body Transcriptome Database Version 1.0. Invest Ophthalmol Vis Sci 60, 3236-3246, doi:10.1167/iovs.19-27106 (2019).

23 Zanotelli, M. R. et al. Stable engineered vascular networks from human induced pluripotent stem cell-derived endothelial cells cultured in synthetic hydrogels. Acta Biomater 35, 32-41, doi:10.1016/j.actbio.2016.03.001 (2016).

24 Curcio A.C, J. M. in Retina Vol. Vol. 1, Part 2: Basic Science and Translation to Therapy, (ed SriniVas R. Sadda Stephen J. Ryan, David R. Hinton, Andrew P. Schachat, SriniVas R. Sadda, C.P. Wilkinson, Peter Wiedemann, Andrew P. Schachat) Ch. 20, 465-481 (London: Elsevier. 5th ed., 2013).

25 Michaelides, M., Hunt, D. M. \& Moore, A. T. The genetics of inherited macular dystrophies. J Med Genet 40, 641-650, doi:10.1136/jmg.40.9.641 (2003).

26 Corominas, J. et al. Whole-Exome Sequencing in Age-Related Macular Degeneration Identifies Rare Variants in COL8A1, a Component of Bruch's Membrane. Ophthalmology 125, 1433-1443, doi:10.1016/j.ophtha.2018.03.040 (2018).

27 Miyagishima, K. J. et al. In Pursuit of Authenticity: Induced Pluripotent Stem Cell-Derived Retinal Pigment Epithelium for Clinical Applications. Stem Cells Transl Med 5, 1562-1574, doi:10.5966/sctm.2016-0037 (2016).

28 Booij, J. C., Baas, D. C., Beisekeeva, J., Gorgels, T. G. \& Bergen, A. A. The dynamic nature of Bruch's membrane. Prog Retin Eye Res 29, 1-18, doi:10.1016/j.preteyeres.2009.08.003 (2010). 
92229 Strunnikova, N. V. et al. Transcriptome analysis and molecular signature of human retinal pigment 923 epithelium. Hum Mol Genet 19, 2468-2486, doi:10.1093/hmg/ddq129 (2010).

92430 Anderson, D. H. et al. Local cellular sources of apolipoprotein E in the human retina and retinal 925 pigmented epithelium: implications for the process of drusen formation. Am J Ophthalmol 131, 926 $927 \quad 31$ 767-781, doi:10.1016/s0002-9394(00)00961-2 (2001).

31 Johnson, L. V. et al. Cell culture model that mimics drusen formation and triggers complement activation associated with age-related macular degeneration. Proc Natl Acad Sci U S A 108, 1827718282, doi:10.1073/pnas.1109703108 (2011).

$930 \quad 32$

Vadlapatla, R. K., Vadlapudi, A. D. \& Mitra, A. K. Hypoxia-inducible factor-1 (HIF-1): a potential target for intervention in ocular neovascular diseases. Curr Drug Targets 14, 919-935, doi:10.2174/13894501113149990015 (2013).

Mammadzada, P., Corredoira, P. M. \& Andre, H. The role of hypoxia-inducible factors in neovascular age-related macular degeneration: a gene therapy perspective. Cell Mol Life Sci 77, 819-833, doi:10.1007/s00018-019-03422-9 (2020).

93634 Chilov, D. et al. Induction and nuclear translocation of hypoxia-inducible factor-1 (HIF-1): 937 heterodimerization with ARNT is not necessary for nuclear accumulation of HIF-1alpha. J Cell Sci 112 ( Pt 8), 1203-1212 (1999).

93935 Wavre-Shapton, S. T., Tolmachova, T., Lopes da Silva, M., Futter, C. E. \& Seabra, M. C. Conditional 940 ablation of the choroideremia gene causes age-related changes in mouse retinal pigment epithelium. PLoS One 8, e57769, doi:10.1371/journal.pone.0057769 (2013).

94236 Huang, J. D., Presley, J. B., Chimento, M. F., Curcio, C. A. \& Johnson, M. Age-related changes in 943 human macular Bruch's membrane as seen by quick-freeze/deep-etch. Exp Eye Res 85, 202-218, doi:10.1016/j.exer.2007.03.011 (2007). 
94537 Spilsbury, K., Garrett, K. L., Shen, W. Y., Constable, I. J. \& Rakoczy, P. E. Overexpression of vascular 946 endothelial growth factor (VEGF) in the retinal pigment epithelium leads to the development of choroidal neovascularization. Am J Pathol 157, 135-144, doi:10.1016/S0002-9440(10)64525-7

948 (2000).

94938 Sun, L. et al. Advanced glycation end products promote VEGF expression and thus choroidal 950 neovascularization via Cyr61-PI3K/AKT signaling pathway. Sci Rep 7, 14925, doi:10.1038/s41598017-14015-6 (2017). 

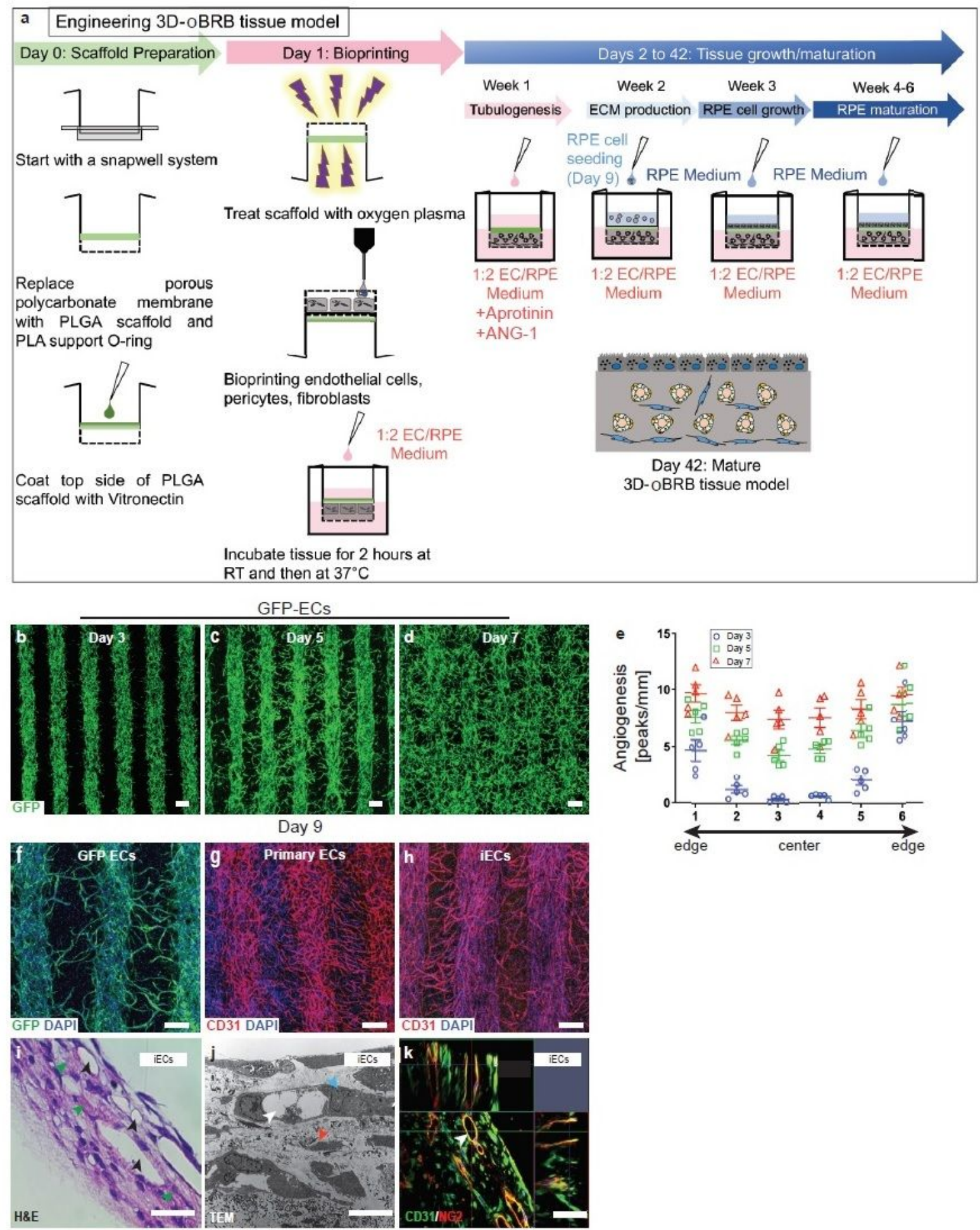

\section{Figure 1}

Design of 3D-oBRB. a, Bioprinting workflow with 93 human endothelial cells (ECs) and RPE. b-d, Vascular development of GFP expressing primary ECs on day 3 (a), day 5 (b) and day 7 (c) after printing. Scale bars, $500 \mu \mathrm{m}$. e, Angiogenesis between printed stripes $(n=5)$. \#p<0.05 in day 3 vs. day $5,+p<0.05$ in day 5 
vs. day $7,{ }^{\star} p<0.05$ in day 3 vs. day 7. f-h, Vascular formation of GFP-positive ECs (green, f), primary ECs (CD31 - red, g), and iPSC-derived ECs (iECs, CD31 - red, h) and nuclei (blue). Scale bars, $500 \mu \mathrm{m}$. i, H\&E images of $10 \mu \mathrm{m}$ thick cross section of iECs derived vascular tissue (day 7). Vasculature is marked with black arrowheads, and ECM components are marked with green arrowheads. Scale bar, $50 \mu \mathrm{m}$.

j, Transmission electron microscope (TEM) images of iECs derived vascular tissue at day 7 . White arrowhead shows a capillary, blue arrowhead marks pericytes, and red arrowhead labels fibroblasts.

Scale bar, $300 \mathrm{~nm}$. k, Orthogonal views of confocal images of $100 \mu \mathrm{m}$ thick tissue sections stained with CD31 (ECs; green) and NG2 (pericytes; red). White arrowheads mark patent EC-derived capillaries. Scale bar, $50 \mu \mathrm{m}$. Statistical significance was attributed to values of $p<0.05$ as determined by two-way ANOVA and Bonferroni post-hoc pair comparison. All error bars indicate STE. 

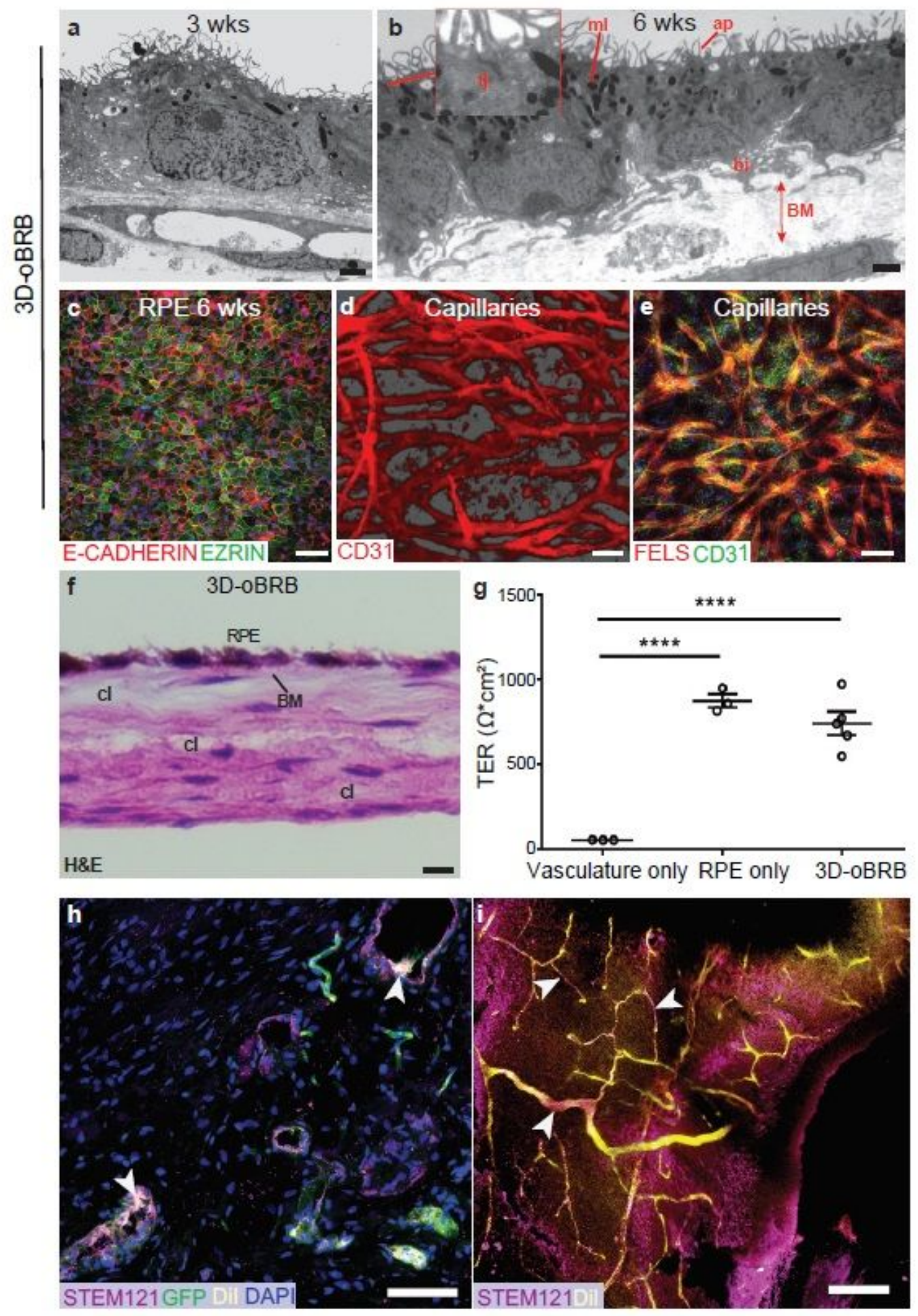

\section{Figure 2}

Engineering of 3D oBRB. a,b, TEM images 209 of 3D-oBRB maturity at week 3 (a) and 6 (b) with RPE pigmentation: melanin (ml), RPE apical processes (ap), RPE basal infoldings (bl), tight junction (tj) formation and Bruch's membrane (BM). Scale bars, $1 \mu \mathrm{m}(\mathrm{a}), 2 \mu \mathrm{m}(\mathrm{b})(\mathrm{n}=3) \mathrm{c}$, Immunostaining for RPE maturity markers E-CADHERIN (red) and EZRIN (green) in 6-week-old tissues. Scale bar, $50 \mu \mathrm{m}$. d, $3 \mathrm{D}$ rendered image of CD31 (red) immunostained capillary-bed in 6-week-old 3D-oBRB. Scale bar, 50 $\mu$ m. 
$(n=4)$ e, Immunostaining for capillary-maturation marker FELS (red) co-labled with EC marker CD31 (green) of confocal images of vascular networks labeled with CD31 (red). Scale bar, $50 \mu \mathrm{m} .(\mathrm{n}=3) \mathrm{f}$, H\&E staining of 6-week-old tissues containing capillaries (cl), RPE, and Bruch's membrane (BM). Scale bar, $10 \mu \mathrm{m} . \mathrm{g}$, Transepithelial resistance (TER) of 3D-oBRB compared to vascular and 2D-RPE $(n=3) . h$, GFP signal in human ECs and immunostaining with STEM121 (magenta) detect human capillaries that are perfused with Dil (yellow) in $10 \mu \mathrm{m}$ cryosections of rat choroid transplanted with 3D-oBRB. Scale bar $75 \mu \mathrm{m}$. ( $\mathrm{n}=8$ eyes from 4 animals). i, Immunostaining for STEM121 (magenta) detects human capillaries integrated with rat capillaries detected by Dil (yellow) perfusion in $100 \mu \mathrm{m}$ vibratome sections of transplanted rat choroid. Scale bar $100 \mu \mathrm{m}$. Statistical significance was attributed to values of $p<0.05$ as determined by one-way ANOVA with Tukey's multiple-comparisons test. ${ }^{\star \star \star \star} p<0.0001$, All error bars indicate STE. 

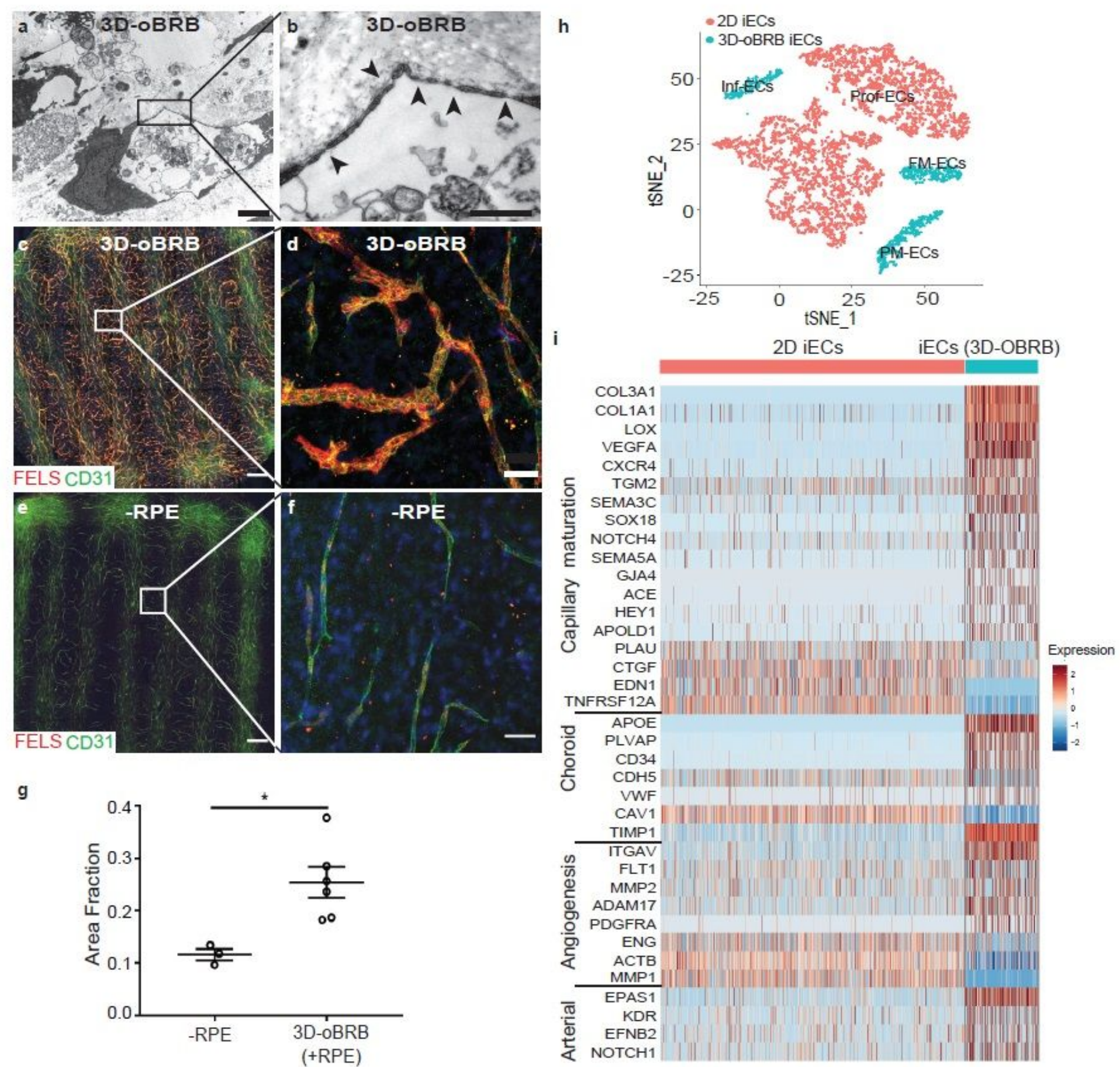

\section{Figure 3}

Capillary maturation in 277 3D-oBRB. a,b, TEM images highlight fenestration (arrowheads) in iECs with in 3D-oBRB capillaries at week 6 . Scale bars, $2 \mu \mathrm{m}(\mathrm{a}), 500 \mathrm{~nm}(\mathrm{~b}) .(\mathrm{n}=3)$. $c-f$, Immunostaining for FELS (red) and CD31 (green) in 3D-oBRB (with RPE; C, d) or just the vasculature (without RPE; e, f). Scale bars, 500 $\mu \mathrm{m}(\mathrm{c}, \mathrm{e})$ and $50 \mu \mathrm{m}(\mathrm{d}, \mathrm{f}) . \mathrm{g}$, Area fraction of FELS and CD31 expression in vascular regions with or without RPE. $(n=3)$. h, TSNE plots from sc-RNA seq of 2D iECs and iECs from 3D-oBRB. i, Gene expression differences between 2D iECs and iECs from 3D-oBRB for genes related to endothelial maturation, choroid, angiogenesis, and arterial development. Statistical significance was attributed to values of $P<0.05$ as 
determined by unpaired t-test. Data depicts results from $n=3012$ cells (2D RPE), $n=4380$ cells (3D-oBRB RPE), $n=5369$ cells (2D iECs), and $n=1294$ cells (3D-OBRB iECs). ${ }^{*} p<0.05$, All error bars indicate STE.
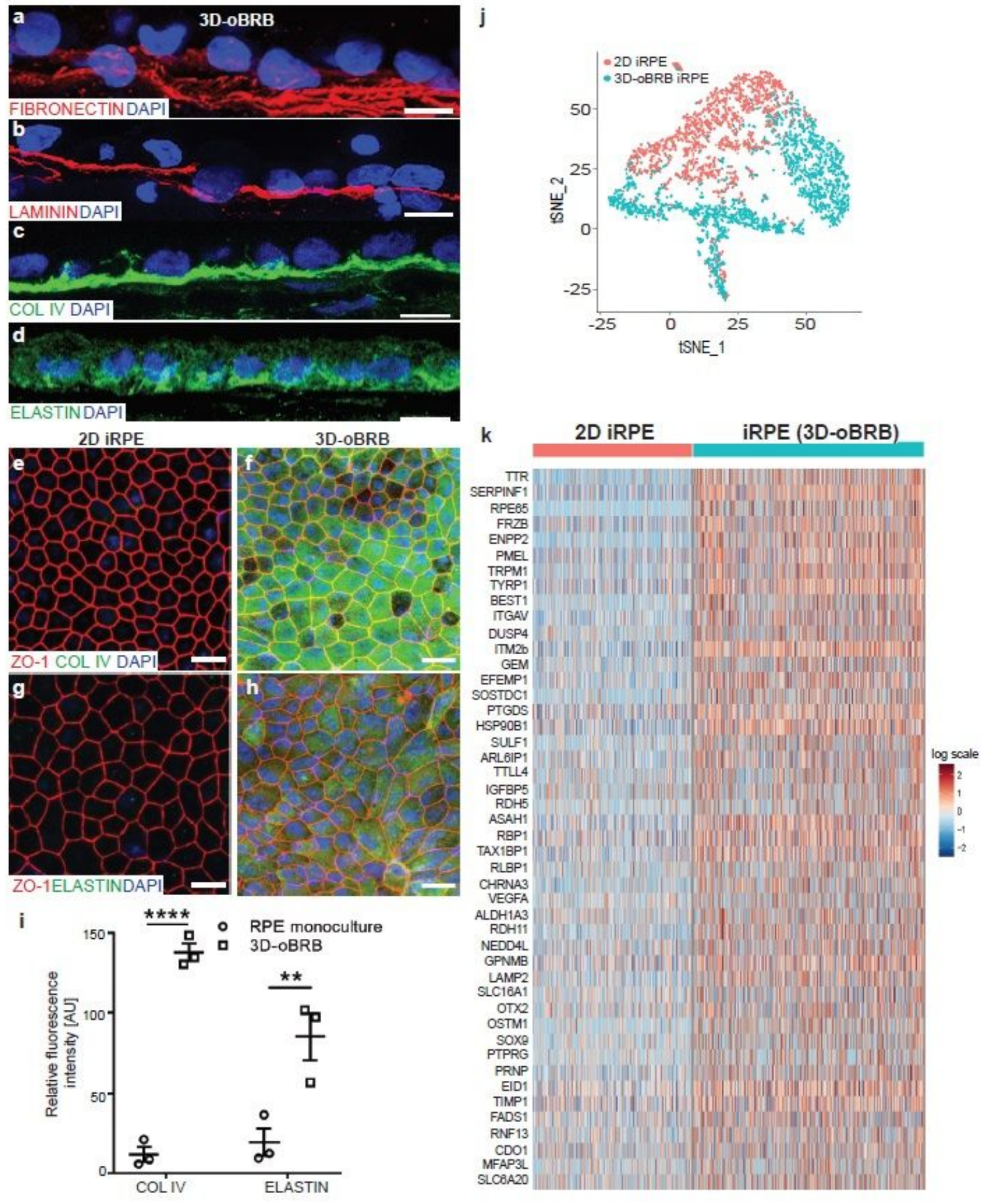

\section{Figure 4}

RPE maturity in 3D-oBRB. a-d, Cross sections of 3D-oBRB immunostained for Bruch's membrane proteins FIBRONECTIN (a), LAMININ (b), COLLAGEN IV (COL IV) (c), and ELASTIN (d). Nuclei stained with DAPI. Scale bars, 10 $\mu$ m. e-h, 2D RPE monoculture $(e, g)$ and 3D-oBRB $(f, h)$, co immunostained for COL IV 
(green) or ELASTIN with ZO-1 (red), and nuclei (blue). Scale bars, $30 \mu \mathrm{m}$. i, Fluorescence mean intensity comparison of ELASTIN and COL IV immunostaining in 2D-iRPE and 3D oBRB models. $(n=3)$. j, tSNE plots from sc-RNA seq of 2D-iRPE and RPE in 3D-oBRB. k, Gene expression of RPE signature genes, comparison between 2D-iRPE and RPE in 3D-oBRB. Statistical significance was attributed to values of $p<0.05$ as determined by two-way ANOVA and Sidak's multiple comparison test. ${ }^{* \star} p<0.01,{ }^{\star \star \star \star *} p<0.0001$. All error bars indicate STE.

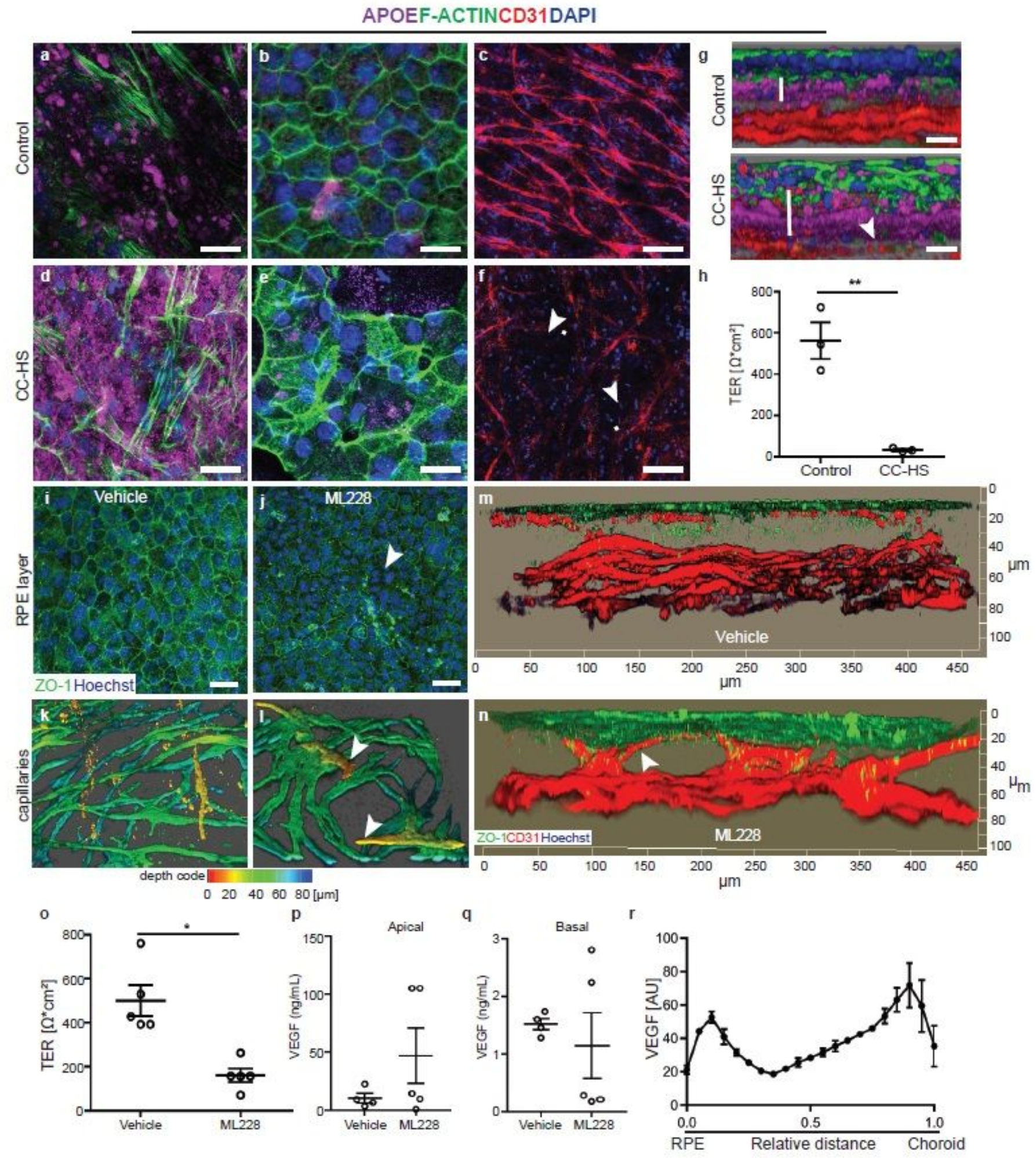

Figure 5 
RPE dependent 392 choroid degeneration in dry and wet AMD models of 3D-oBRB. a-g, En face (a-f), cross-section (g) views of APOE (magenta), CD31 (red) immunostaining, F-ACTIN (green), Hoechst (blue) in complement competent human serum (CC-HS) treated and control 3D-oBRB. Arrowheads mark degenerated RPE and capillaries. Scale bars, $100 \mu \mathrm{m}$. (g) white vertical lines mark Bruch's membrane. Scale bars, $10 \mu \mathrm{m}$. ( $n=3)$ h, TER of control and CC-HS treated 3D-oBRB $(n=3)$. i,j, Confocal images of RPE from 3D-oBRB, immunostained with ZO-1 (green) and Hoechst (blue). Arrowhead marks lost ZO-1 signal. Scale bars, $30 \mu \mathrm{m}$. k,l, Depth code of 3D reconstructed confocal images. Color gradient along z-axis (100 $\mu \mathrm{m}$ depth) starting from subRPE zone (red) to the end of choroid (blue); in vehicletreated 3D-oBRB $(\mathrm{k})$ and in ML228 treated 3D-oBRB (I). Arrowheads mark capillaries in the sub-RPE region. Scale bars, $100 \mu \mathrm{m}$. m,n, Side view images of CNV, immunostained with ZO-1 (green) and

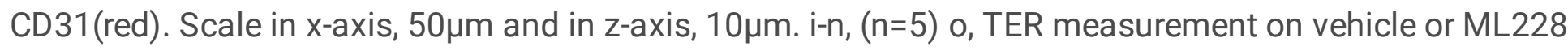
treated 3D-oBRB $(n=5)$. p, q apical ( $p)$ and basal $(q)$ VEGF secretion in vehicle and ML228 treated 3DoBRB $(n=4)$. r, Fluorescence intensity of VEGF staining in cryosectioned 3D-oBRB tissue slice.

Quantification of subRPE region ( 0.0 fractional distance) to the bottom of the choroid layer ( 1.0 fractional distance) $(n=3)$. Statistical significance was attributed to values of $p<0.05$ as determined by paired t-test $(o, p, q)$ or two-way ANOVA and Sidak's multiple comparison test $(\mathrm{g}, \mathrm{h}) .{ }^{*} \mathrm{p}<0.05,{ }^{* \star *} \mathrm{p}<0.001,{ }^{* \star \star *} \mathrm{p}<0.0001$. All error bars indicate STE 408 (g,o,p,q,r). 

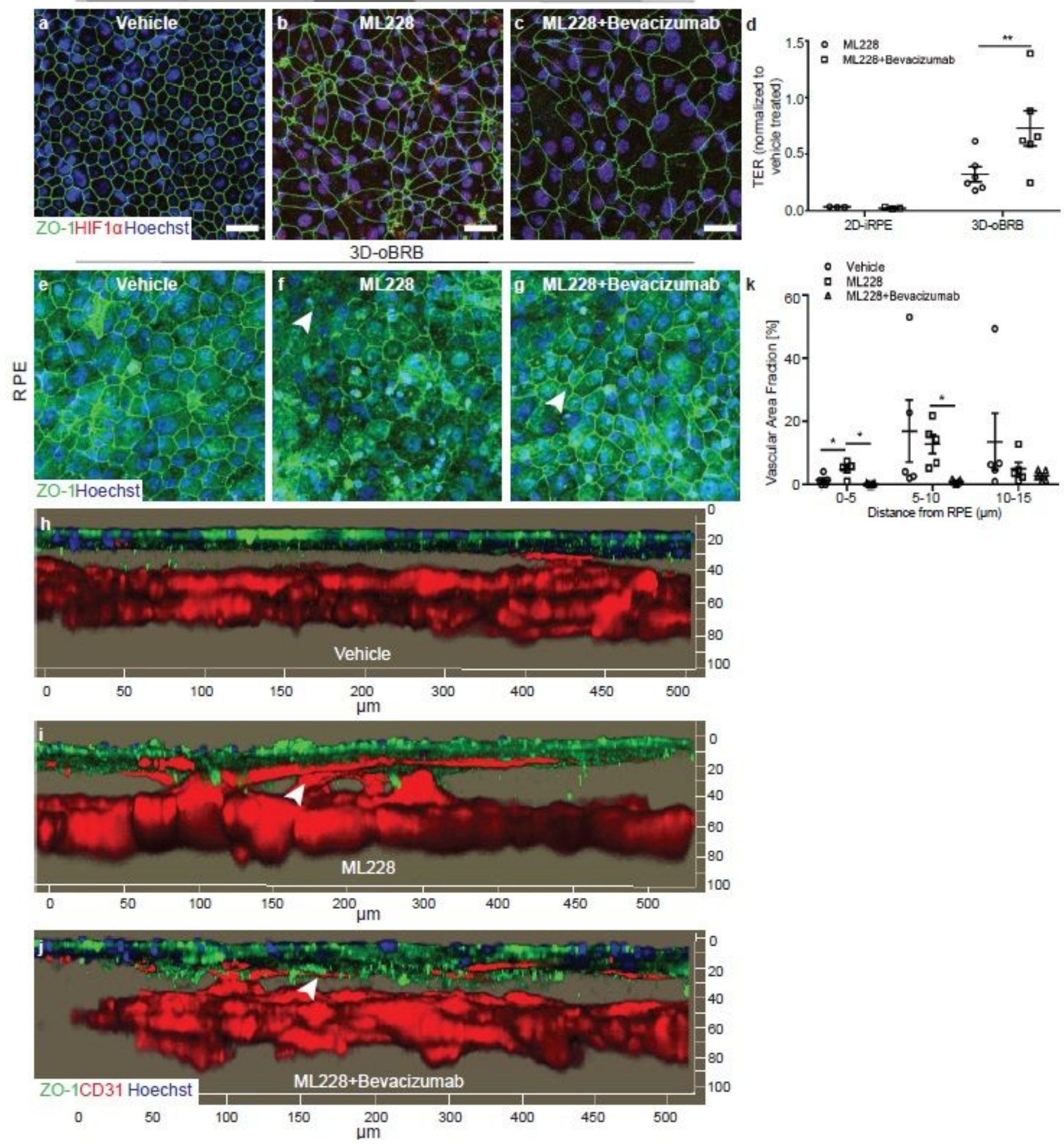

\section{Figure 6}

Bevacizumab treatment suppresses 438 wet-AMD in 3D-oBRB. a-c, RPE monoculture at 2 weeks treated with DMSO as a vehicle control (a), ML228 $(2 \mu \mathrm{M})(\mathrm{b})$, and ML228 $(2 \mu \mathrm{M})+$ bevacizumab $(0.284 \mathrm{mg} / \mathrm{ml})(\mathrm{c})$, immunostained for HIF-1a (red), ZO-1 (green), and nuclei stained with Hoechst (blue). Scale bars, 30 $\mu \mathrm{m}$. $(n=3) . d$, TER measurement comparison between 2D-iRPE and 3D-oBRB for ML228 amd ML228+bevacizumab treated samples. TER values were normalized to vehicle treated 3D-oBRBs. (2D- 
iRPE, $n=3 ; 3 D-o B R B, n=6$ ). e-g, Maximum intensity projection images of RPE of 3D-oBRB, immunostained with ZO-1 (green) and stained with Hoechst for nuclei (blue). Degenerated and recovered RPE are marked with arrowheads in ( $f$ and g). Scale bars, $25 \mu \mathrm{m}$. h-j, Side view of 3D reconstructed images of vehicle $(\mathrm{h})$, ML228 (i), and ML228+bevacizumab (j) treated 3D-oBRB tissues, immunostained with ZO-1 (green) and CD31(red). Arrowheads in (i) mark hyperproliferating capillaries and in (j) mark retracted capillaries. Scale in $x$-axis, $50 \mu \mathrm{m}$ and in z-axis, $10 \mu \mathrm{m}$. e-j, $(n=4) . k$, Vascular area fraction was calculated from CD31 positive area in each z-stack. $(n=5)$. Statistical significance was attributed to values of $p<0.05$ as determined by unpaired t-test $(\mathrm{m})$ or two-way ANOVA and Sidak's multiple comparison test $(\mathrm{d}, \mathrm{h}, \mathrm{l}) .{ }^{*} \mathrm{p}<0.05,{ }^{* \star} \mathrm{p}<0.01,{ }^{\star \star \star *} \mathrm{p}<0.0001$. All error bars indicate STE.

\section{Supplementary Files}

This is a list of supplementary files associated with this preprint. Click to download.

- SupplementrayV1.mp4

- SupplementrayV2.mp4

- SupplementrayV3.mp4

- SupplementrayV4.mp4

- SupplementrayV5.mp4

- SupplementrayV6.mp4

- Supplementarytablesandfigures.pdf

- flatNMETHA44557Anreditorialpolicychecklist.pdf

- flatNMETHA44557Anrreportingsummary.pdf 